\title{
Philosophiques
}

\section{Les études platoniciennes : contribution canadienne (1970-1977)}

\section{Yvon Lafrance}

Volume 4, numéro 1, avril 1977

URI : https://id.erudit.org/iderudit/203063ar

DOI : https://doi.org/10.7202/203063ar

Aller au sommaire du numéro

Éditeur(s)

Société de philosophie du Québec

ISSN

0316-2923 (imprimé)

1492-1391 (numérique)

Découvrir la revue

Citer cet article

Lafrance, Y. (1977). Les études platoniciennes : contribution canadienne (1970-1977). Philosophiques, 4(1), 51-99. https://doi.org/10.7202/203063ar d'utilisation que vous pouvez consulter en ligne.

https://apropos.erudit.org/fr/usagers/politique-dutilisation/ 


\title{
BULLETIN
}

\section{LES ÉTUDES PLATONICIENNES : CONTRIBUTION CANADIENNE (1970-1977)}

\author{
par Yvon Lafrance
}

L'excellence et le nombre des ouvrages publiés, dans les dernières années, sur la philosophie de Platon par des collègues canadiens justifient, à notre avis, que nous présentions aux lecteurs de la revue ce bulletin sur la contribution canadienne aux études platoniciennes. Cette contribution comprend sept ouvrages parus entre 1970-1977 et un nombre assez impressionnant d'articles publiés dans des revues scientifiques nationales et internationales'. Pour ne pas alourdir outre mesure notre examen critique, nous nous limiterons ici à la seule revue des ouvrages. Dans l'ensemble nous croyons qu'ils constituent une contribution valable à l'avancement des études platoniciennes et qu'ils obéissent tous aux plus hautes exigences de la recherche scientifique.

D'une façon générale, il existe trois genres d'études sur Platon. Les unes se consacrent à une interprétation d'ensemble de la philosophie de Platon, d'autres se limitent à l'étude d'une notion, d'un thème, d'un problème ou d'une théorie, enfin un certain nombre d'études abordent un dialogue particulier. Aucun platonisant canadien n'a encore donné son interprétation d'ensemble des dialogues platoniciens. Par contre, quatre des ouvrages que nous examinerons appartiennent au second genre d'études: l'ouvrage de T'M. Robinson porte sur le problème de l'Âme', celui de L. Paquet sur le thème du Regard', tandis que l'ouvrage de R.A.

1. Voir notre appendice à la fin de cet article. Nous prenons le terme "canadien " au sens de toute personne qui travaille dans une institution canadienne, à l'exception de L. Brisson qui est canadien mais travaille au CNRS (Paris).

2. ROBINSON, T.M., Plato's Psychology, University Press of Toronto, 1970 (repr. 1972), 202 $\mathrm{PP}$.

3. PAQLIEI, L., Platon. La Médiation du Regard, Essai d'interprétation, Leiden, E.J. Brill, 1973. $484 \mathrm{pp}$. 
Shiner aborde le problème de la connaissance et de la réalité dans le Pbilèbe et celui de R.E. Allen le problème de la théorie des Formes dans l'Eutbyphron'. Quant au troisième genre d'études il est représenté par l'ouvrage de P. Seligman sur le Sophiste ${ }^{6}$, celui de L. Brisson sur le Timée et la traduction ainsi que le commentaire du Phédon par D. Gallop ${ }^{8}$. Nous commencerons cette revue critique par les ouvrages qui traitent de problèmes pour la terminer par ceux qui abordent un dialogue particulier. Á l'intérieur de chaque groupe nous adopterons dans notre présentation l'ordre chronologique des dialogues.

\section{T.M. Robinson et le problème de l'Âme}

Le titre de l'ouvrage de Robinson doit sans doute être pris au sens étymologique du terme "psychology" puisque, de toute évidence, il ne s'agit pas d'une étude complète sur la psychologie de Platon, mais plus précisément d'une analyse du terme " psyché " dans les dialogues de Platon. L'auteur nous avertit dans sa Préface (p. vii) que trois monographies seulement ont été consacrées à ce sujet: celle de A.Éd. Chaignet (De la Psychologie de Platon, 1862), qu'il considère marquée par l'idéalisme philosophique, celle de J. Simson (Der Begriff der Seele bei Platon, 1889), non suffisamment élaborée, et celle de H. Barth (Die Seele in der Phi. losophie Platons, 1921) qu'il trouve entachée des préjugés de la Lebens philosophie. Cependant, comme il apparaît inévitable pour toute étude sur Platon, le sujet n'est pas neuf et il suffit d'ouvrir le Lustrum (1960-1965, pp. 340-382) pour remarquer la quantité d'études qui ont été récemment consacrées au problème de l'âme chez Platon. L'auteur a choisi pour sa part de s'en tenir à l'analyse du texte platonicien et a relégué dans les notes au bas des pages la mention de ces études de sorte que le lecteur ne doit pas s'attendre à trouver dans cet ouvrage l'état actuel des recherches platoniciennes sur le problème de l'âme. Avant de procéder à des remar-

4. SHINER, R.A., Knowledge and Reality in Plato's Philebus, Assen, Van Gorcum \& Comp. B.V., 1974,79 pp.

5. ALLEN, R.E., Plato's Eutbyphro and the Earlier Theory of Forms, New York, Humanities Press, 1970, 171 pp.

6. SELIGMAN. P., Being and Not-Being. An Introduction to Plato's Sophist, The Hague, Martinus Nijhoff, 1974, $120 \mathrm{pp}$.

7. BRISSON. L., Le Même et l'Autre dans la Structure ontologique du Timée de Platon, Paris, Klincksieck, 1974, $589 \mathrm{pp}$.

8. GAlLOP. D., Plato Pbaedo, Oxford, Clarendon Press, 1975, 245 pp. 
ques critiques particulières, nous aimerions tenter de dégager la doctrine platonicienne de l'âme à laquelle aboutit cette analyse du terme " psyché " en nous servant de la conclusion de l'ouvrage (pp. $158.163)$.

La doctrine platonicienne de l'âme pose quatre problèmes fondamentaux : ceux de la nature de l'âme, de sa relation avec le corps, de sa simplicité et de son immortalité. L'analyse du terme "psyché " dans les dialogues permet à l'auteur de soulever d'une façon non systématique chacun de ces problèmes.

En ce qui concerne la nature de l'âme, celle-ci est considérée dans les dialogues socratiques de plusieurs façons : l'âme est principe cognitif, principe éthique, source d'identité personnelle ou encore une "counter-person "dans le mythe du Gorgias, comme d'ailleurs dans tous les mythes. Tous ces sens se retrouvent dans le Phédon qui ajoute par ailleurs deux autres sens : l'âme comme principe de vie et comme une sorte de fluide qui se répand dans tout le corps et auquel l'auteur donne l'étrange appellation biologique d'ectoplasme. Ces divers sens du Phédon se retrouvent également dans la République où la confusion entre l'âme comme principe de vie et l'âme comme principe moral entraînerait, selon Robinson, de sérieux doutes sur la validité de l'argumentation du premier livre. Dans le Timée, l'âme cosmique est purement noétique et garde une analogie avec la partie rationnelle de l'âme humaine telle que décrite en République, IV. Le Phèdre qui, selon Robinson, suit chronologiquement le Timée, présenterait un réel progrès sur le Timée en ce sens qu'il considère l'âme comme source de mouvement, mais encore ici cette âme est conçue seulement comme noétique. Le mythe du Politique présente une heureuse combinaison des vues du Timée et du Phèdre. Comme dans le Timée, l'âme du monde est contingente et le Démiurge non contingent. Mais les données du Phèdre ont mené Platon à décrire le Démiurge comme jouissant d'un mouvement éternel de sorte que l'automotricité attribuée à l'âme devient d'une nature spécifique. Le Philèbe met en parallèle l'âme du monde et l'âme individuelle en faisant de l'âme du monde la source de l'âme individuelle. Cette âme du monde est essentiellement une matrice de l'intelligence, elle est contingente et apparaît comme la contrepartie du Démiurge du Timée et du Politique. Les Lois élargissent la conception de l'âme jouissant d'une automotricité éternelle et en font une 
conception de l'âme comme substance cosmique (cosmic-stuff) plutôt que simple principe cognitif. L’âme, en tant que substance cosmique, devient la condition sine qua non de toute activité intellectuelle et morale. L'Epinomis reprend les vues des Lois, mais parle aussi des activités démiurgiques qu'il essaie d'identifier avec l'âme rationnelle du monde du Timée et peut-être même avec le Démiurge. Cette nomenclature des divers sens du terme "psyché " révèle à elle seule la difficulté pour l'interprète de Platon d'arriver à une conception systématique de la nature de l'âme individuelle et cosmique.

On se retrouve devant des difficultés analogues lorsqu'on pose le problème de la relation âme-corps. Les dialogues socratiques partagent à ce sujet des vues différentes : pour le Protagoras, le corps est purement une possession de l'âme, dans l'Alcibiade I, il est à la fois une possession et un instrument de l'âme tandis que dans le Charmide, le corps est conçu comme une partie inaliénable de l'âme à la manière d'une relation biologique entre la partie et le tout. Par contre, dans le Phédon, la relation âme-corps est conçue selon le plus pur dualisme. Le corps est nuisible, sinon mauvais, les plaisirs et les désirs du corps sont distingués de ceux, plus nobles, de l'âme de sorte que celle-ci doit être soumise au processus libérateur de la purification. Dans la République, l'âme, qui s'identifie au moi, entretient une relation biologique avec le corps comme dans le Charmide ou encore est conçue comme une sorte de SuperEgo qui transcende le composé âme-corps. Dans le Timée, l'âme individuelle est située dans une partie particulière du corps tandis que l'âme cosmique, après avoir reçu le mouvement du Démiurge, le communique au corps du monde. Le Pbilèbe affirme l'origine psychique des plaisirs et des peines que le Phédon avait tendance à rapporter au corps. Dans les Lois, l'âme-substance, comme l'âme du monde du Timée, est antérieure au corps du monde, elle est la contrepartie du Démiurge du Timée et du Politique ainsi que de l'intelligence transcendante du Philèbe.

L'âme humaine est-elle simple ou composée? La réponse de Platon à cette question est loin d'être uniforme. Le Gorgias et le Ménon fournissent certaines indications en faveur d'une composition de l'âme tandis que le Pbédon, en mettant une affinité entre l'âme et l'Idée considère l'âme comme quelque chose de statique, d'homogène et d'indivisible. Pourtant l'analogie politique de la 
République conduit Platon à la théorie de la tripartition de l'âme de sorte que le conflit âme-corps du Pbédon se situe à l'intérieur même de l'âme entre ses diverses parties. Le Timée conserve cette tripartition de l'âme individuelle, mais Robinson soutient que la division de l'âme en partie mortelle et partie immortelle semble plus essentielle que la tripartition. Le Phèdre revient à la division tripartite de la République, IV. Le Politique décrit un élément irrationnel dans l'âme du monde contrairement au Timée qui conserve la rationalité à l'âme du monde pour rejeter l'irrationalité du côté de la Nécessité. Les Lois et l'Epinomis reviennent à la bipartition de l'âme, ce qui, selon Robinson, représenterait un retour à la conception populaire de la bipartition de l'âme.

Platon a consacré un dialogue entier au problème de l'immortalité de l'âme et c'est le Phédon. Robinson affirme cependant que ces preuves de l'immortalité de l'âme se trouvent viciées par l'ambiguîté même de la notion d'âme. Le livre X de la République introduit une nouvelle preuve de l'immortalité de l'âme humaine, celle de l'automotricité de l'âme, mais l'âme dont il s'agit n'admet pas de parties tandis que le livre IV du même dialogue développe une théorie de la tripartition de l'âme. Dans le Timée, l'unique partie de l'âme qui jouit de l'immortalité est la partie rationnelle. Le Phèdre suit le Timée sur ce point en soutenant que l'âme n'est pas contingente, mais éternelle, et, selon Robinson, cette propriété ne peut appartenir qu'à l'âme noétique. Ici le Phèdre transfère à l'âme individuelle et cosmique les propriétés que le Timée attribuait au Démiurge. Mais du même coup, le Phèdre soutient aussi une tripartition de l'âme de sorte qu'on pourrait être autorisé à conclure à l'immortalité des trois parties de l'âme. Ce qui serait contraire au Timée.

On le voit bien, les problèmes que pose une analyse philologique et chronologique du terme de "psyché » dans les dialogues platoniciens demeurent d'envergure. Robinson fournie dans son ouvrage des données textuelles, mais à partir de ces données il est impossible de dégager une doctrine cohérente de l'âme chez Platon comme le montre la conclusion de cette analyse philologique. L'ouvrage de Robinson nous incline à croire qu'il n'existe pas de doctrine cohérente de l'âme chez Platon. Mais du coup cette constatation nous apparaît étrange. Nous concevons difficilement que Platon ait conçu l'âme et le corps comme un tout biologique, 
comme deux substances séparées ou comme un Super-Ego transcendant sans que ces modifications dans sa conception du rapport âme-corps ne doivent recevoir une explication que l'auteur ne donne pas. Ou encore que Platon ait oscillé toute sa vie entre la simplicité de l'âme et sa composition, qu'il soit passé cavalièrement de l'une à l'autre sans que l'interprète puisse nous expliquer le pourquoi de ces constantes oscillations. Ou encore qu'un même dialogue, la République, comprenne une doctrine de la simplicité de l'âme et en même temps une doctrine de la composition de l'âme. On a reproché à Robinson de n'avoir pas développé suffisamment une approche philosophique des problèmes". Nous croyons le reproche partiellement justifié. Non pas que nous pensions ici qu'une analyse purement conceptuelle aurait pu permettre à Robinson de résoudre ces problèmes d'incohérence dans la doctrine platonicienne de l'âme. Lorsque nous pensons à une approche philosophique, nous nous référons au souci d'intégrer la donnée textuelle dans le contexte d'un problème où elle prend son sens véritable en devenant l'élément d'une doctrine en voie d'élaboration. Pour notre part nous ne sommes pas sûr qu'un strict traitement unilatéral de nature philologique puisse donner toute sa portée philosophique à un texte. Ces données doivent être constamment rapportées à la problématique propre du dialogue, au degré d'adhésion de Platon à l'argumentation générale ainsi qu'à l'évolution d'ensemble de la pensée de Platon. Le travail de l'interprète est de chercher la cohérence des textes, surtout lorsqu'ils se rapportent à une même oeuvre, ou à une même période d'élaboration de l'oeuvre, et c'est bien à son corps défendant qu'il doit abandonner la lutte pour conclure à l'incohérence d'une pensée philosophique. L'ouvrage de Robinson nous révèle les limites de la méthode philologique d'une façon d'autant plus évidente que l'interprète manie cette méthode avec une habileté remarquable et qu'elle se présente dans son interprétation de Platon sous son jour le meilleur.

En refusant ainsi à son analyse philologique des données textuelles relatives à l'âme de lui fournir un fondement solide pour une éventuelle doctrine cohérente de la psyché chez Platon, Robinson ne discute pas les modèles d'interprétation proposés par les platonisants contemporains. Dans la mesure où le commentaire et

9. DYBIKOWSK1. J. Critical Notice : T.M. Robinson, Plato's Psychology, ds. Can. Journ. of Philosophy. 3 (1973), 131. 
l'interprétation constituent un travail d'équipe, il est du plus haut intérêt pour l'avancement des études platoniciennes de critiquer ces modèles ou d'en proposer un meilleur. Par exemple, en ce qui concerne la théorie des parties de l'âme, deux modèles fondamentaux ont été proposés pour résoudre les apparentes contradictions du texte platonicien ${ }^{10}$. Le premier, que nous appellerions spéculatif, ne fait pas appel à l'évolution de la pensée de Platon, mais à deux points de vue différents. Ainsi Zeller, Archer-Hind et Shorey ont soutenu que l'âme est simple comme l'enseigne le Pbédon et qu'elle se réduit au logistikon de la République et au principe im. mortel du Timée lorsque Platon la considère en elle-même et dans sa nature essentielle. Mais lorsque Platon considère l'âme dans son état d'union au corps, il la conçoit comme composée de parties, c'est-à-dire selon un modèle de bipartition entre le logistikon et l'alogistikon qui se transforme parfois en tripartition. Ainsi Platon aurait soutenu à la fois la simplicité et la composition de l'âme selon deux points de vue différents : celui de l'âme désincarnée et celui de l'âme incarnée. Ce modèle spéculatif se réfère à la problématique du dialogue. Le second modèle essaie de résoudre l'apparente contradiction entre la simplicité de l'âme et sa composition en recourant à l'évolution de la pensée de Platon. Ici toutes les hypothèses ont été proposées. Selon Pfeiderer et Rohde, Platon aurait d'abord adhéré à la thèse de la tripartition de l'âme humaine ( $R e ́$ publique, IV, Phèdre), puis serait passé à la simplicité (République, $\mathrm{X}$, Phédon) pour revenir à la tripartition (Timée). Selon Dümler, l'évolution se serait faite plutôt en sens inverse : Platon serait parti d'abord de la simplicité de l'âme (République I, X, Gorgias) pour passer ensuite à la tripartition (Phèdre, République, IV) et revenir à la simplicité (Ménon, Phédon, République X). Selon Schultess, Leissner, Groag, Platon aurait d'abord soutenu la thèse de la simplicité de l'âme (Phédon), puis celle de sa tripartition (Phèdre), et finalement aurait affirmé la simplicité de l'âme désincarnée et la composition de l'âme incarnée (République $\mathrm{X}$, Timée). À la place de cette évolution en trois phases, on peut aussi penser à une évolution en deux phases comme le soutiennent Frutiger et Robin. Platon aurait d'abord soutenu la simplicité de l'âme (Phédon) pour adopter d'une façon définitive la tripartition de l'âme (République, Pbèdre, Timée). On aura remarqué ici un

10. (In pourra se référer pour tout ce qui suit à l'ouvrage de P. Frutiger, Les $M$ ythes de Platon, (1930), New York, Arno Press, 1976, pp. 76-96. pp. 125-143, pp. $211-225$. 
élément commun à toutes ces interprétations de la théorie de l'âme chez Platon : certains passages platoniciens soutiennent la simplicité de l'âme, d'autres passages sa composition. L'interprète peut donc partir de ce consensus pour tenter d'expliquer cette apparente contradiction. Et ce travail ne relève pas de la simple analyse philologique, mais de la spéculation philosophique. Cette spéculation philosophique n'est pas pour nous une pure analyse conceptuelle qui couperait le texte de son contexte, et le contexte de l'ensemble de l'évolution de la pensée de Platon, mais une coordination des textes en fonction de leur contexte qui est la problématique propre de chaque dialogue avec ses différents niveaux de langage, littéraire, mythique et dialectique ainsi qu'une référence constante de ces textes à une évolution de la pensée de Platon. On pourrait faire des remarques analogues en ce qui concerne la théorie de l'immortalité de l'âme. Doit-on la considérer comme une théorie que Platon n'a jamais vraiment pris au sérieux comme l'ont soutenu Teichmüller et Couturat?'S'agit-il au contraire d'une doctrine que Platon a pris au sérieux, mais qu'il aurait toujours considérée comme une simple croyance comme le soutenait Robin? Ou doiton croire avec Frutiger que la théorie de l'immortalité de l'âme était pour Platon une simple croyance fondée sur une tradition religieuse orphico-pythagoricienne avant de devenir avec le Phédon une doctrine que Platon considérait comme scientifiquement certaine? On ne peut discuter sérieusement de l'immortalité de l'âme chez Platon sans tenir compte de ces questions et des différents niveaux de langage du texte platonicien aussi bien que du degré d'adhésion de Platon lui-même à son propre langage. Pour ce travail, les instruments philologiques demeurent déficients, il faut les utiliser en fonction de la spéculation philosophique.

Nous venons de souligner l'importance que l'on doit accorder à la problématique propre de chaque dialogue platonicien ainsi qu'à l'évolution de la pensée de Platon dans la spéculation philosophique portant sur les apparentes contradictions du texte de Platon. Depuis Schleiermacher les platonisants contemporains s'accordent pour fonder cette évolution de la pensée de Platon sur l'ordre chronologique des dialogues. Tout récemment, J.N. Findlay a remis en question l'idée de Schleiermacher selon laquelle l'ordre chronologique des dialogues représente l'évolution de la pensée de Platon, au nom des doctrines orales de Platon $w^{11}$. Mais ces doctrines orales

1. FINDIAY, J.N., Plato. The Written and Umwritten Doctrines, London, Routledge \& Kegan Paul, New York, Humanities Press, 1974, p. 29. 
demeurent si controversées qu'on doit s'attendre, à moins de découvertes sensationnelles sur le plan des manuscrits, à ce que pour longtemps notre représentation de l'évolution de la pensée de Platon ne trouve pas de base plus solide que l'ordre chronologique des dialogues. Or, Robinson adopte dans son ouvrage un ordre chronologique assez répandu dans la tradition anglo-saxonne et qui consiste à considérer le Timée comme un dialogue de maturité de Platon qui suivrait immédiatement la République et précéderait le Phèdre. Robinson appuie cet ordre chronologique sur un article de G.E.L. Owen". qui, à notre avis, a été suffisamment réfuté par H.F. Cherniss', G.C. Field, G. Vlastos et d'autres platonisants ${ }^{1.1}$ pour nous autoriser à maintenir le Timée parmi les dialogues de vieillesse et par conséquent postérieur au Phèdre. Nous voyons difficilement comment la seule analyse stylistique de Billig qui constitue une pièce maîtresse de l'argumentation d'Owen peut renverser les conclusions d'un siècle de recherches et qui situent le Timée parmi les derniers dialogues de Platon. Sur ce point les recherches de Lutoslawski, de Reader, de Ritter et de Wilamowitz coïncident et elles nous apparaissent définitives ${ }^{15}$. Quant aux arguments d'Owen fondés sur le paradigmatisme du Parménide et l'opposition entre la " genesis" et "l'ousia " présente dans le Timée et les dialogues de maturité, mais complètement absente dans le Sophiste, le Pbilèbe et le Parménide, il faudrait, pour les accepter, avoir traversé la critique serrée qu'en a fait H.F. Cherniss. On verrait alors qu'ils ne tiennent pas le coup devant une analyse approfondie des dialogues de vieillesse et de maturité. Une troisième série d'arguments se fonde sur la ressemblance entre certaines théories politiques et psychologiques du Timée et de la Républi. que. Cherniss n'aborde pas ces arguments dans sa critique, mais l'on sait très bien en suivant les recherches sur la chronologie effectuées au siècle dernier que ce genre d'arguments spéculatifs a souvent conduit les chercheurs à des conclusions que l'on reconnaît unanimement aujourd'hui comme erronées. S'il y a une leçon que les platonisants ont dégagée de cette aventure chronologique, c'est

12. OWEN, G.E.L.. The Place of the Timaeus in Plato's Dialogues. (1953), ds. Studies in Platsis Metaplysics, ed. by R.E. Allen, London, Routledge \& Kegan Paul, New York, Humanities Press, 1965. pp. 313-338.

13. CHERNISS . H.F. The Relation of the Timaeus to Plato's Later Dialogues, (1957), Ibid. pp. $339-378$.

14. Voir Lustrum (1959-Bd. 4) pp. 65-67

15. Pour la commodité, on pourra consulter sur ce point le tableau que donne David Ross dans son ouvrage Plato's Theory of Ideas, Oxford, Clarendon Press, 1951, p. 2. 
bien celle qui nous enseigne à considérer les arguments spéculatifs comme ambivalents dans ce genre de recherches. À l'intérieür de certaines limites, on peut les organiser pour justifier l'une ou l'autre de deux hypothèses chronologiques contraires. Par ailleurs, et cela Cherniss l'avait déjà observé dans son article en réponse à celui d'Owen, nous pensons que ce déplacement du Timée vers les dialogues de maturité a obéi à un préjugé philosophique des analystes d'Oxford qui voyaient d'un très bon oeil la disparition de la théorie des Formes dans les dialogues postérieurs au Parménide. Cela leur permettait de présenter plus facilement un Platon qui avait abandonné ses rêveries mystico-ontologiques pour se consacrer à des problèmes plus fonctionnels de logique et d'analyse du langage ${ }^{16}$.

Nous ne croyons pas que Robinson accepte ce préjugé philosophique qui a sans doute motivé le remaniement chronologique récent du Timée. Mais si nous avons insisté sur ce point, c'est que nous croyons que cette thèse chronologique de G.E.L. Owen devrait être définitivement abandonnée et qu'une doctrine platonicienne de l'âme ne peut être reconstruite qu'en considérant le Timée postérieur à la République et au Phèdre. C'est ainsi que beaucoup de jugements de Robinson qui se fondent sur cet ordre chronclogique erroné portent à faux et ne peuvent être retenus.

Ces remarques critiques n'enlèvent cependant rien à la valeur indubitable de l'ouvrage de Robinson. Nous croyons au contraire que sa lecture est indispensable pour quiconque s'intéresse au problème de l'âme chez Platon. Nous déplorons seulement que l'auteur se soit arrêté à l'analyse philologique du terme " psyché "sans tenter de résoudre les apparentes contradictions auxquelles le conduisait cette analyse. Et s'il s'était aventuré sur cette voie philosophique, il aurait pu arriver à des conclusions difficilement acceptables à cause de l'ordre chronologique qu'il adopte pour le Timée $e^{16}$. C'est aussi à l'intérieur de cette tradition philologique

16. Bien que Cherniss ne cite aucune étude à l'appui de cette constatation, nous nous permettons nous-même de renvoyer le lecteur aux articles de G. Ryle, "Plato's Parmenides " (I) (II), ds Mind. 48 (1939) 129-151, 302-325, R. Robinson, "Forms and Etror in Plato's Theaete tus, "ds. The Pilos. Rev. 59 (1950) 3-30, R.C. Cross, Logos and Forms in Plato (1954), ds. Studies in Plato's Metaphysics, op. cit. pp. 13-31, D.W. Hamlyn, "The Communion of Forms and the Development of Plato's Logic", ds. The Philos. Rev. 5 (1955) 289-302.

16a. Nous signalons aux lecteurs les comptes rendus suivants de l'ouvrage: Y. Brès, Ret. Et. Gr., 83 (1970) 260-262, J.V. Luce, Hermath., 11 (1971) 91-92, J. Philip, Dialogue, 10 (1971) 347-349, G. Santas, Pbilos. Rev., 81 (1972) 244-246, M.A. Stewart, Philos. 
que nous situerions l'étude de L. Paquet sur le thème du Regard che\% Platon.

\section{L. Paquet et le thème du Regard}

L'étude du terme "psyché " nous référait à un problème fondamental du platonisme, celle du terme $\beta \lambda \dot{\epsilon} \pi \epsilon \nu$ et de ses composés nous introduit au platonisme par le biais de l'expression esthétique. En effet, le regard ne constitue pas un problème philosophique chez Platon, mais plutôt un leitmotiv littéraire qui, de l'avis de L. Paquet, revêt cependant une importance philosophique (p. 17). C'est pourquoi l'ouvrage de L. Paquet se divise en deux parties: une partie philologique (chap. I-VIII) et littéraire (chap. IX) et une partie philosophique (chap. X-XIV).

Le point de départ de cette étude est un passage du Timée (28a-b) dans lequel il est dit que le Démiurge travaille les yeux sans cesse fixés sur un modèle éternel et qui, pour cette raison, produit nécessairement une oeuvre belle. Ce thème du regard, fait remarquer L. Paquet, se retrouve dans certains textes majeurs où l'artisan devient un point de comparaison constant: dans le Gorgias ( $503 \mathrm{e}-\mathrm{d}$ ), l'homme vertueux est comparé aux peintres, aux architectes, aux constructeurs de navires, aux médecins parce qu'il travaille les yeux fixés sur un modèle pour produire de beaux discours ; dans le Cratyle (389a-390e), c'est le nomothète qui travaille les yeux fixés sur le nom en soi pour produire l'oeuvre belle qu'est le langage humain; dans la République (VI, 500c-501b), c'est le gouvernant-philosophe qui travaille à la manière d'un peintre sur la toile des caractères humains en imitant le modèle divin les yeux fixés sur la justice en soi (p. 23). À partir de ces textes, L. Paquet se croit autorisé à mener son enquête dans l'ensemble des dialogues de Platon et à remonter au-delà de Platon jusqu'aux temps homériques. Ce qui nous vaut une histoire litté-

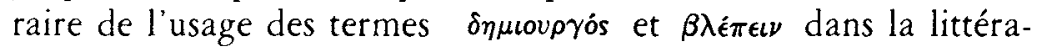
ture grecque préplatonicienne. Cette première partie philologique et littéraire de l'ouvrage nous apparaît la plus solide et la moins

Qtatt. 2! (1971) 172.73, G. Anagnostopoulos, Journ. Hist. Pbilos., 10 (1972) 217-21. A. Graeser, Gnomon, 43 (1972) 342-46, J. Dybikowski, Can. Journ. Pbilos. 3 (1973. 1974) 131-142, P. Louis, Rev. de Pbilol. Littérature et Hist. Anc., 45 (1971) 152-53, J. Moreau, Rez'. Et. Anc., 72 (1970) 424-25, J. Ortall, Crisis, 20 (1973) 425-26. J.B. Skemp, Clast. Ret', 22 (1972) 335-39, R.K. Sprage, Class. Pbilol., 67 (1972) 63-64, D.W. Hamlyn, Pbilos. Books, 12 (1971) 25-26. 
contestable. Elle est suffisamment exhaustive, bien articulée et les conclusions qui s'en dégagent reposent sur des analyses de textes tout à fait remarquables. Cependant l'intention de L. Paquet va beaucoup plus loin. Dans la mesure où son enquête philologique le mène au coeur des grandes théories cosmologiques, ontologiques, épistémologiques et artistiques du platonisme, il soutient que le thème du regard ne touche pas seulement à l'expression littéraire de la philosophie platonicienne, mais à son contenu proprement philosophique. Il montre d'abord que la répétition de la formule

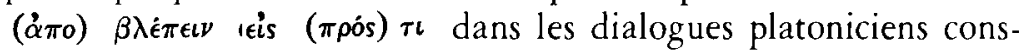
titue un cas typique de ce que Des Places appelait naguère une formule de récurrence ${ }^{17}$ (pp. 205-254). On retrouve celle-ci au moment stratégique des dialogues, toujours intimement reliée au contenu philosophique de la discussion. De plus, le regard ne réfère pas seulement au but visé par l'artisan, mais aussi au modèle sur lequel il fixe les yeux en travaillant, ce qui relie le regard à la théorie des Formes intelligibles (pp. 254-268), qu'il s'agisse du regard de l'artisan (pp. 269-296), de l'initié (pp. 296-356) ou encore de l'artiste (pp. 357-415). Dans cette seconde partie de son ouvrage que nous croyons plus sujette à la critique que la première partie, L. Paquet fait reposer sa compréhension du platonisme sur deux notions : la notion de transposition qu'il emprunte à $\mathrm{A}$. Diès ${ }^{18}$ et la notion d'analogie qu'il utilise abondamment à la suite de $\mathrm{P}$. Grenet ${ }^{19}$. Ces notions ont été couramment utilisées dans le platonisme français contemporain et nous dirons plus loin ce que nous en pensons ${ }^{20}$. Pour le moment, il nous suffit de noter que, pour L. Paquet, le fait que Platon se serve des termes $\delta \eta \mu$ iovoós et $\dot{\alpha} \pi 0^{-}$ $\beta \lambda \epsilon \epsilon \epsilon \iota \nu$, qui véhiculaient dans la culture de l'époque un sens péjoratif pour le premier et plutôt banal pour le second, pour les appliquer à l'activité noble du Fabricant de l'Univers, du législateur ou de l'artiste, constitue un cas de transposition. Cette transposition entraîne évidemment des analogies, c'est-à-dire des comparaisons entre plusieurs termes et réalités du type : le Démiurge est à l'Univers ce qu'est l'ouvrier à l'égard de son oeuvre ou encore le gouvernant-philosophe est à la cité ce qu'est le peintre à sa toile.

17. DES PLACES, E., Une formule platonicienne de récurrence. Paris, Les Belles Lettres, 1929.

18. DIES. A. Autour de Platon. Essais de critique et d'histoire, Paris, Beauchesne, 1927.

19. CRENET. P.. Les origines de l'analogie pbilosophique dans les dialogues de Platon. Paris. Vrim, 1948.

20. Voir à ce sujet notre article: "L'Usage de l'analogie dans l'interprétation de Platon." ds. Didegute $15(1976) 282-289$. 
Nous retrouvons ici le sens proprement grec d'analogie, celui de proportion (pp. 10-11), c'est-à-dire d'égalité de rapports entre quatre termes si l'on veut se reporter à la définition géométrique d'Euclide et qui s'exprime par la formule générale : $A / B=$ C/D". L. Paquet semble avoir été frappé par l'usage abondant de ces analogies chez Platon. Il en arrive ainsi à penser que ce vocabulaire traduit une intuition fondamentale du platonisme, à savoir, que la réalité entière est conçue par Platon comme un véritable “ jeu de proportions" (pp. 427-428). Et c'est ici sans doute que se situe l'idée directrice et le fil conducteur de tout l'ouvrage de $\mathrm{L}$. Paquet. Mais il nous faut revenir sur la partie philologique et la partie philosophique de l'ouvrage pour en dégager les articulations principales et proposer quelques remarques critiques.

Puisque le regard du Démiurge constitue le point de départ de cette étude, il était naturel que l'auteur ouvre son enquête philologique par l'examen de l'usage du terme "démiurge " dans la littérature préplatonicienne (pp. 25-41) et dans les dialogues platoniciens (pp. 42-65). Les textes homériques utilisent le terme sans aucune connotation préjorative. Ce n'est, semble-t-il, qu'à la suite des mouvements sociaux de la Grèce du VIIe siècle au Ve siècle qui amènent la répartition des classes sociales sur la base des métiers, que le terme acquiert une connotation péjorative et tend d'ailleurs à être remplacé par les termes $\beta \dot{\alpha} \nu \alpha v \sigma o s, x \epsilon \iota p \bar{\omega} \nu \alpha \xi$, et

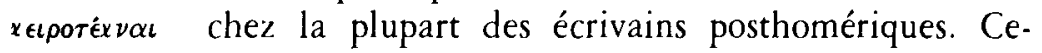
pendant il y a deux exceptions à cette chute du $\delta \eta \mu \iota v \rho \gamma$ ós, de l'ouvrier en général, dans l'échelle de valeurs des écrivains posthomériques: il s'agit de la tradition des médecins hippocratiques qui référent le terme "démiurge " au beau et noble travail $\mathrm{du}$ " chirurgien " et du "plasticien " et d'un texte des Mémorables de Xénophon $(I, 4,2)$ que $L$. Paquet soupconne d'être inspiré de Platon (p. 39-41) et qui réfère le terme au "Démiurge " de l'Univers. Chez Platon, le terme $\delta \eta \mu$ ovo ${ }^{\circ}$ ós se distingue du terme $\beta \dot{\alpha} \nu$ avoos; : dans la majorité des cas il utilise le premier terme dans le sens d'une activité physique ou non physique caractérisée par l'intelligence, l'ordre et la beauté tandis qu'il réserve le second terme pour désigner une activité physique qui dégrade l'âme et qui s'oppose à la paideia. L'auteur aborde ensuite l'étude du terme $\beta \lambda \epsilon \dot{\epsilon} \epsilon \iota \nu$ dans la littérature préplatonicienne: d'abord sous sa forme simple

21. EUCILIDE, Élintents, V, déf. 6. 
(pp. 71-94), ensuite les formes à préverbe (pp. 95-108), puis suivi

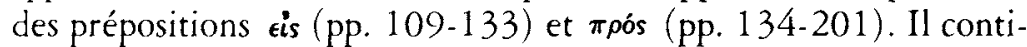
nue son enquête avec le verbe $\dot{\alpha} \pi \circ \beta \lambda \dot{\epsilon} \pi \epsilon \boldsymbol{\nu}$ suivi de $\epsilon$ s (pp. 153 . 176 ) et de $\pi \rho$ ós (pp. 177-201). L'auteur avoue avoir consulté pour cette enquête philologique environ vingt-neuf témoins (pp. 250, n. 1). Celle-ci nous permet de passer en revue toutes les nuances du verbe $\beta \lambda \dot{\epsilon} \pi \epsilon \iota \nu$ et de dégager en ce qui concerne l'usage du verbe suivi des prépositions eis et $\pi$ oós, le bilan suivant. L'expression impose l'idée d'un objet de valeur, impressionnant, prestigieux, séduisant qui détermine certaines attitudes du regard : l'espoir, la supplication, le désir, la menace et l'intimidation (pp. 149.

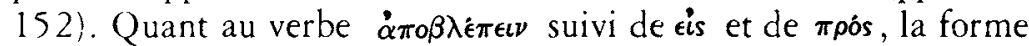
demeure relativement neuve au moment où Platon l'utilise et le sens physique du regard tend à céder le pas au sens du regard de l'esprit. De plus, l'objet visé représente toujours une valeur à laquelle le sujet se réfère comme à une norme (pp. 198-200).

Ce bilan philologique de la littérature préplatonicienne conduit L. Paquet à examiner l'usage de la formule $(\dot{\alpha} \pi o) \beta \lambda \dot{\epsilon} \pi \epsilon \nu$ cis ( $\pi \rho o s) \tau \iota$ dans le dialogue platonicien. Ce qui nous vaut un chapitre admirable sur la forme littéraire du dialogue dans lequel l'auteur réussit par touches successives à décrire le mouvement général du dialogue, à situer les personnages, à marquer les moments importants de la discussion. Le thème du regard apparaît dès lors comme un véritable leitmotiv littéraire, c'est-à-dire selon l'expression bien connue de $\mathrm{E}$. des Places, une formule de récurrence. Celle-ci apparaît à titre indicatif pour ponctuer les moments importants d'une discussion, soit pour attirer l'attention des interlocuteurs du dialogue sur une valeur, soit pour souligner l'objet même du dialogue ou encore l'importance d'un argument, soit encore pour suggérer, selon le langage de V. Goldschmidt, l'Appel à l'Essence. Ce chapitre bien construit et articulé s'inspire des meilleures pages de La Question Platonicienne de R. Schaerer, du moins en ce qui concerne son approche du dialogue comme drame philosophique.

Toute cette première partie de l'ouvrage de L. Paquet relève en réalité de l'histoire littéraire. On a reproché à l'auteur de n'avoir pas fait une approche socio-économique de la figure du Démiurge dans le Timée ${ }^{22}$. L'intérêt d'une telle approche est sans

22. BRISSON. L., Compte rendu de louvragre paru dans Dialogue, 15 (1976) 159-160. 
doute indubitable et les pages que L. Brisson consacre, dans un ouvrage que nous analyserons plus loin, au Démiurge du Timée montrent la fécondité d'une approche socio-économique. Cependant on ne saurait nier également l'intérêt d'une approche littéraire du terme onprovopós qui permet au lecteur de cheminer à travers toutes les oeuvres importantes de la littérature grecque. Il s'agit pour nous de deux méthodes complémentaires qui, si elles étaient pratiquées simultanément, nous révéleraient sans doute un lien étroit entre la littérature d'une époque et son contexte socioéconomique. Cependant on peut comprendre aussi que les deux méthodes puissent être pratiquées séparément à moins qu'on ne veuille présupposer que toute l'histoire des oeuvres de l'esprit humain se réduit en dernière instance à une histoire socioéconomique ${ }^{23}$. On a aussi reproché à l'auteur d'avoir utilisé dans cette partie philologique de son ouvrage une méthode trop intuitive qui ne tient pas suffisamment compte des champs sémantiques, de l'analyse syntaxique et de la rhétorique ${ }^{24}$. Cette critique, à notre avis, demeure pertinente en soi dans la mesure où elle fait appel à l'usage de méthodes linguistiques plus raffinées dans la recherche philologique. Mais du coup on doit remarquer que l'usage de ces méthodes conduit ordinairement à des monographies linguistiques, comme le prouve l'ouvrage relativement récent de J. Lyons ${ }^{25}$, alors que le projet de L. Paquet était, semble-t-il, de rédiger une monographie philosophique sur des fondements philologiques. Les méthodes ne sont pas des absolus et leur choix dépend ordinairement du genre de travail qu'on se propose de faire. Nous croyons, pour notre part, que la méthode utilisée par L. Paquet est suffisante pour donner un fondement philologique raisonnable à ses réflexions philosophiques ultérieures. On ne demande pas à un baigneur dans une piscine d'utiliser un télescope pour apercevoir le ballon qu'on lui lance, même s'il est en train de nager dans une piscine ultramoderne.

Ceci nous amène à la seconde partie de l'ouvrage. Le thème du regard qui avait été traité dans les chapitres précédents d'un

23. Nous ne croyons pas d'après son ouvrage sur le Timée que L. Brisson adopterait d'emblée ce présupposé. Ce qui devrait laisser place, nous semble-t-il, dans son esprit à une distinction entre l'histoire littéraire d'un terme et son histoire socio-économique. Nous notons par ailleurs que les deux enquêtes aboutissent à donner au terme " démiurge " les mêmes significations au cours de son évolution historique.

24. LEROIIX, G., Étude critique de l'ouvrage parue dans Pbilosopbiques, 2 (1975) 278-284.

25. LYONS, J., Structural Semantics. An Analysis of Part of the Vocabulary of Plato, Oxford, Blackwell, 1972. 
point de vue philologique et littéraire sert maintenant à nous introduire au coeur des théories fondamentales du platonisme. Pour L. Paquet, le regard n'est pas seulement dans le dialogue platonicien une formule de récurrence littéraire, mais aussi une formule de récurrence doctrinale : on le retrouve à l'intérieur de la théorie cosmologique (le Démiurge regarde un modèle), politique (le législateur regarde le juste en soi), épistémologique (le philosophe regarde les Formes intelligibles), esthétique (l'artiste regarde le beau en soi) et morale (le vertueux regarde le meilleur). Toutes ces activités disparates ont cet élément commun d'impliquer un regard sur un modèle de sorte que le regard constitue un véritable médiateur qui les rend toutes analogiques. Ce sont ces analogies que $\mathrm{L}$. Paquet se propose de nous faire découvrir dans la seconde partie de son ouvrage.

En s'appuyant sur les textes d'Alcibiade I (130-135) ${ }^{26}$ et des Lois XII (960-966), L. Paquet nous suggère de considérer le "regard " comme une analogie polyvalente qui comprend à la fois la visée de l'artisan vers un but précis qui est "le meilleur ", la vision de l'initié de l'objet transcendant et le regard de l'artiste qui est simultanément visée et vision d'un Modèle (p. 268). Chacun des éléments de cette analogie se trouve étudié dans les chapitres subséquents : la visée de l'artisan (pp. 269-295), la vision de l'initié (pp. 296-356) et la vision de l'artiste (pp. 357-415). L'artisan est présenté par Platon comme un spécialiste qui vise toujours le meilleur et qui produit une oeuvre belle, intelligente et ordonnée. Cette conception de l'activité rationnelle de l'artisan sert à caractériser les activités les plus diverses et les plus disparates : l'activité du législateur des noms (Cratyle, 389-390), celles du législateur politique (Rép. IV, 421b4), de l'orateur authentique (Gorg. $504 \mathrm{~d} 6$ ), de l'homme vertueux (Prot. 316c), du citoyen (Gorg. $504 \mathrm{~d}$ ), voire même de l'État tout entier (Rép. IV, 433d-434c). Il existe ainsi entre ces diverses activités une analogie, c'est-à-dire une ressemblance en ce sens qu'elles sont toutes décrites comme étant la visée du meilleur en vue de produire une oeuvre belle. Le regard devient ainsi un médiateur entre ces différentes forme d'activité en les reliant les unes aux autres et nous introduit à un contenu doctrinal : la hantise fondamentale, chez Platon, de la ra-

26. L. Paquet ne souligne malheureusement pas les problèmes relatifs à l'authenticité de l'Alcibiade I. 
tionalité et de la compétence dans l'activité humaine (p. 295). Mais dès lors cette " visée " de l'artisan implique la "vision " d'un Modèle, ce qui nous amène au coeur de la dialectique platonicienne et de la théorie des formes intelligibles. Dans cette foulée, l'auteur passe en revue les textes classiques sur la dialectique : les exposés du Banquet, de la République, VI-VII et du Phèdre où il montre le rôle important que joue le "regard " à l'intérieur de ces textes. En effet, l'image du regard implique les éléments essentiels de l'ascension dialectique : la purification du regard qui se détache de la multiplicité, la conversion de l'oeil intérieur vers la réalité stable, l'unification de la vision dans l'unité de l'être (p. 355). Bref, dans ces textes le regard renvoie à un objet transcendant c'est-à-dire à la réalité intelligible. L'artiste, par ailleurs, se présente comme la synthèse de l'artisan et de l'initié en ce sens qu'il produit d'après ce qu'il a vu, ce qui correspondrait par ailleurs, selon $\mathrm{L}$. Paquet, à la réalité du milieu où l'on ne distinguait plus entre l'artisan et l'artiste (p. 357). Ici les mêmes analogies reviennent sous la plume de l'interprète de Platon : le législateurartiste, le philosophe-artiste, le démiurge-artiste, l'orateur-artiste, le nomothète-artiste. Tous ces personnages sont reliés entre eux par une activité dont la norme est la Forme intelligible. Cette relation de l'artiste aux Formes intelligibles est décrite en terme de regard de sorte que celui-ci constitue l'élément médiateur qui permet de comparer entre elles ces diverses activités et d'établir les analogies suivantes :

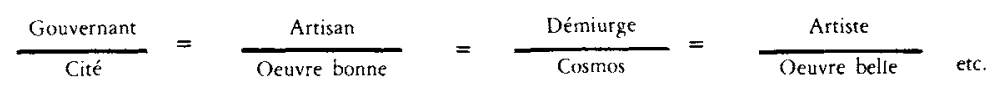

L'articulation générale de cette partie philosophique de l'ouvrage nous semble beaucoup moins nette que celle de la première partie. À l'intérieur de chacun de ces chapitres, l'auteur utilise une quantité de textes des dialogues platoniciens sans avoir pris le soin de les ordonner en fonction de leur valeur respective par rapport à la thèse qu'il défend. Souvent les mêmes textes reviennent au cours d'un même chapitre ou sont exploités dans les trois chapitres sans compter leur retour dans le dernier chapitre. Ce qui crée une impression désagéable de répétitions inutiles et de piétinements sur place. Pour notre part, nous aurions aimé cheminer à travers ces chapitres selon un plan plus rigoureux. Si l'auteur avait réussi ce travail d'ordonnance rigoureuse de ses textes, il aurait ainsi pu dire 
ce qu'il avait à dire en beaucoup moins de pages. On a reproché à l'auteur d'avoir utilisé les notions de transposition et d'analogie ${ }^{27}$. Mais cette critique ne nous semble pas justifiée. Le terme " trans. position" n'est pas un terme vague comme l'affirme L. Brisson, mais il sert à décrire des situations fort précises du discours platonicien. Il faudrait relire ici les pages que L. Paquet consacre à l'ascension dialectique du Banquet (pp. 296-328) pour se convaincre de l'utilité de ce terme dans quelques cas d'interprétation du texte platonicien. Platon se sert ici de certains éléments des mystères d'Éleusis, abandonnant certains autres qu'il juge sans valeur pour construire son discours philosophique. Les quatre degrés d'ascension dialectique, la présence du guide, l'époptie finale sont des éléments que Platon retient de l'expérience religieuse des mystères d'Éleusis alors qu'il élimine du discours philosophique tout le côté kermesse de ces rites. C'est ce passage d'éléments qui appartenaient d'abord à l'expérience religieuse et qui sont récupérés selon des choix préalables dans le discours philosophique que L. Paquet, à la suite de A. Diès, appelle une transposition. Ainsi peut-on dire que l'ascension dialectique du Banquet est une transposition au plan philosophique de l'expérience religieuse vécue par les initiés aux mystères d'Éleusis. Même chose dans le Phèdre en ce qui concerne la rhétorique sophistique et la rhétorique philosophique. Quant au terme " analogie ", l'interprète de Platon peut difficilement l'éviter puisque comme le disait jadis J. Wahl : "l'analogie... c'est tout Platon ". Il est possible que L. Paquet ait manqué de rigueur dans l'emploi trop abondant qu'il fait du terme en n'indiquant pas de façon précise les éléments de l'analogie, mais les nombreux exemples qu'il nous en fournit semblent suffisants pour nous convaincre de son utilité dans l'interprétation du platonisme. Ce qu'il faut comprendre ici c'est que, pour L. Paquet, Platon a conçu la réalité comme une grande oeuvre d'art avec ses jeux de lumière et de proportions et dans laquelle chaque niveau de réalités renvoie à l'autre selon le fameux " canon des proportions " utilisé par les artistes de la Grèce. C'est pourqoi loin d'être une interprétation thomiste, comme l'a soutenu L. Brisson, le Platon de L. Paquet en est une interprétation esthétique. En effet, le lieu à partir duquel L. Paquet parle de Platon n'est pas l'analogie de l'être, mais l'oeuvre d'art

27. Je me réfère ici aux comptes rendus de L. Brisson et de G. Leroux défà cités en notes 22 et 24 . 
dans toute sa splendeur telle que pouvait la concevoir le génie grec de l'époque's.

Les deux ouvrages que nous venons de parcourir couvraient autour d'un problème et d'un thème l'ensemble des dialogues de Platon. Les deux prochains, celui de R.E. Allen et celui de R.A. Shiner nous proposent une réflexion sur la théorie des formes intelligibles dans l'Eutbyphron et le Philèbe respectivement.

\section{R.E. Allen et la première Théorie des Formes}

L'ouvrage de R.E. Allen Plato's Euthyphro and the Earlier Theory of Forms se divise comme l'indique son titre en deux parties. La première comprend une traduction coupée de commentaires d'un dialogue de jeunesse de Platon: l'Eutbyphron. Cette première partie est conçue dans le projet global de l'auteur comme un exemple typique de dialectique socratique, c'est-à-dire d'un procédé de discussion par questions et réponses et qui doit servir de fondement textuel à la seconde partie de l'ouvrage, fondement d'ailleurs que l'auteur se permettra d'élargir au besoin à l'aide des autres dialogues de jeunesse. La seconde partie de l'ouvrage, hautement spéculative, comprend une interprétation originale de la théorie des Formes qui est loin de se limiter aux dialogues de jeunesse mais qui atteint les dialogues de maturité et même les dialogues de vieillesse. En fait, R.E. Allen se propose de nous prouver la présence de deux théories des Formes dans l'oeuvre de Platon. Nous laisserons de côté la première partie de l'ouvrage (pp. 15.65) pour concentrer notre attention sur cette seconde partie (pp. 67. 166).

On pourrait résumer brièvement la thèse que l'auteur défend de la façon suivante. Il existe une première théorie des Formes dans l'Euthyphron et d'autres dialogues socratiques. Cette théorie est différente de celle rencontrée dans le Pbédon et la République parce qu'elle possède un statut ontologique différent (p. IX). En effet, l'intérêt principal des dialogues socratiques ne réside pas dans

28. Nous signalons au lecteur les comptes rendus suivants sur l'ouvrage de L. Paquet: F.X. Druet, Les Et. Class. (1973) 462.463, J. Moreau, Rev. Et. Anc., 76 (1974) 156-157. J. Ortall, Crisis. 22 (1975) 137.138. J. Ferguson, Journ. of Roman Studies, 65 (1975) 201. 202. G. Leroux, Philosophiques, 2 (1975) 253-285, L. Brisson, Dialogue, 15 (1976) 157. 162, Y. Lafrance, Dialogzue, 15 (1976) 282-289, Bulletin de l'Association Guillaume Budé, $35\{1976\} 374-375$. 
les résultats obtenus à travers l'exercice dialectique, mais dans la dialectique elle-même. Or, cette dialectique socratique repose en définitive sur le présupposé ontologique qu'il existe une nature ou une essence des choses, que cette nature ou essence des choses est en même temps une norme qui permet de les juger et un universel qui rend possible leur définition réelle. Ainsi la Forme revêt dans la dialectique socratique un aspect ontologique ou métaphysique qui affecte le cours du monde dans la mesure où les Formes sont les natures réelles des choses et un aspect logique puisqu'elles donnent lieu à des définitions réelles, ou plus précisément à une possibilité de définitions réelles. Mais cette première théorie des Formes ne doit pas être confondue avec celle des dialogues de maturité qui repose sur une ontologie dualiste en faisant intervenir les oppositions bien connues de l'Être et du Devenir, de l'Apparence et de la Réalité, du Repos et du Mouvement, de la Raison et de la Sensation, du Corps et de l'Âme, c'est-à-dire finalement une doctrine des deux mondes, le monde sensible et le monde intelligible (pp. 67-68). On avait coutume jusqu'ici d'expliquer la différence entre la conception des Formes dans les dialogues de jeunesse et celle des dialogues de maturité en ayant recours au témoignage d'Aristote (Met. I, 987b ss., XIII, 1078b18 ss., XIII, 1086b2 ss.), selon lequel les Formes chez Platon sont 'séparées' tandis que dans la définition socratique elles ne le sont pas. R.E. Allen remet longuement en question ce témoignage d'Aristote pour conclure que les Formes socratiques sont aussi séparées que les Formes platoniciennes (pp. 130-145). En effet, les Formes socratiques sont 'séparées' des cas particuliers en ce sens qu'elles ne leur sont pas identiques et qu'elles leur sont antérieures ontologiquement. Où trouver la différence entre ces deux théories des Formes? Elle se trouve uniquement dans la manière de concevoir la séparation ( $p$. 147). Dans les dialogues de maturité la séparation est conçue à la lumière de la doctrine des deux mondes tandis que dans les dialogues de jeunesse elle est conçue à la lumière de l'universel. L'évolution de Platon de la première théorie des Formes à la seconde s'explique, selon R.E. Allen, par les problèmes que posait à Platon la possibilité d'une recherche de la définition réelle à laquelle se vouait la dialectique socratique. Ces problèmes sont soulevés pour la première fois dans le Ménon (80d-e) alors qu'on se demande comment il est possible de chercher une chose que l'on ne connaît pas. Ce paradoxe de la recherche, Platon le solutionnait par sa doc- 
trine de la réminiscence qui, selon l'auteur, impliquait la possibilité d'une connaissance a priori. C'est ainsi que Platon est amené à concevoir, en fonction de cette connaissance a priori, une nouvelle ontologie, celle du monde sensible et du monde intelligible. Dès lors, la théorie des Formes de la maturité constitue un développement naturel de la théorie des Formes des dialogues de jeunesse. D'une certaine façon on peut dire qu'elle est la même parce qu'elle contient la première comme l'une de ses parties, et qu'elle n'est pas la même parce qu'elle repose sur une ontologie différente (pp. 165 166).

Cette thèse, comme il est facile de le voir, va à l'encontre des interprétations communément reçues de la théorie des Formes et de son rôle dans les dialogues platoniciens. Elle va également à l'encontre de notre conception commune de la philosophie socratique. Elle remet en question notre compréhension des témoignages d'Aristote sur lesquels se base en partie notre interprétation de la théorie des Formes. En effet, on considère communément qu'il n'existe pas de théorie des Formes séparées dans les dialogues de jeu-

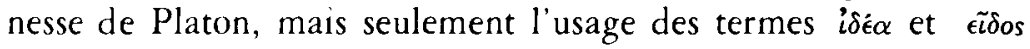
dans le cadre d'une doctrine socratique de la définition ${ }^{29}$. La théorie des Formes séparées n'apparaît que dans les dialogues de maturité de Platon et constitue une doctrine proprement platonicienne. Elle est ensuite critiquée sinon en elle-même, du moins dans les interprétations qu'on pouvait en faire à l'Académie, à partir du Parménide pour subir ensuite des modifications diverses dans les dialogues de vieillesse de Platon. De plus, en soutenant, d'une part, que les dialogues socratiques représentent la doctrine du Socrate historique (pp. 12-13), et, d'autre part, qu'ils contiennent une théorie des Formes séparées, R.E. Allen nous amène à mettre au compte de Socrate une théorie attribuée normalement à Platon ou encore à convertir la méthode socratique de la définition en une théorie des Formes séparées. Puisque le témoignage d'Aristote demeure gênant pour une telle opération, l'auteur se voit forcé de donner au témoignage d'Aristote une interprétation susceptible de correspondre aux vues qu'il se propose de défendre sur la théorie des Formes. La démarche fondamentale de l'auteur, on le voit, va de la spéculation au texte et non pas du texte à la spéculation de sorte que le texte n'est plus lu pour lui-même, mais en fonction de la spéculation.

2\%. ROSS. D., Plato's Theory of Ideas (1951), Oxford, 1963, pp. 11-21. 
Cette thèse de R.E. Allen que nous venons de présenter comme tout à fait nouvelle, ne l'est pourtant pas. Elle a été soutenue au début du siècle par A.E. Taylor dans ses Varia Socratica (1911) et par J. Burnet dans son édition du Phaedo (1911) et son ouvrage Greek Philosophy (1914) pour être ensuite réfutée dans une étude substantielle de L. Robin (1916) ${ }^{30}$. Cependant l'intérêt de l'ouvrage de R.E. Allen vient de ce qu'il essaie d'établir la thèse d'une théorie socratique des Formes séparées sur des fondements nouveaux. Ces fondements constituent une véritable construction spéculative où la dialectique socratique est examinée à l'aide de la notion aristotélicienne de la définition per genus et differentiam (pp. 79-105), de la théorie platonicienne des Formes de la maturité et de notions empruntées à la logique ainsi qu'à l'analyse contemporaine du langage (pp. 105-129) pour aboutir finalement à une réinterprétation du témoignage d'Aristote pour le rendre conforme aux conclusions des précédentes spéculations (pp. 130-145). Certes la construction que nous offre R.E. Allen demeure fascinante dans sa cohérence logique, la rigueur et la profondeur des analyses conceptuelles, l'originalité des points de vue qu'elle soulève. Mais une fois passé ce premier moment de fascination spéculative, nous nous demandons si cette construction brillante correspond vraiment au Socrate historique, ou plus précisément aux textes des dialogues de jeunesse de Platon, des témoignages d'Aristote sur la philosophie de Socrate et celle de Platon sans oublier le Socrate des Mémorables de Xénophon que l'auteur ne cite d'ailleurs que deux fois (p. 12 n. 1, p. 75) et de façon anodine ${ }^{31}$. Nous demeurons également un peu sceptique sur cette construction pure. ment spéculative d'une première théorie socratique des Formes séparées qui consiste à soulever à propos du dialogue socratique des problèmes qui relèvent soit de la philosophie aristotélicienne (pp. 83-87), soit de la philosophie platonicienne (pp. 120-125), soit encore de la philosophie contemporaine du langage (pp. 107 120). En réalité, l'interprète projette sur la dialectique socratique des contenus doctrinaux logiques, ontologiques et linguistiques qui lui sont étrangers.

30. ROBIN, L., "Sur une hypothèse récente relative à Socrate ". ds. Rev. Et. Gr., 29 (1916) 129 ss. Réimprimé dans La Pensée Hellénique der Origines à Épicure, Paris, 1967, pp. 138-176.

31. Nous nous permettons ici de renvoyer le lecteur à notre étude sur la valeur de ces témoignages : Le Problème Socratique, ds. La Philosophie et les Philosophes, Montréal, 1973, pp. $13-26$. 
L'auteur soutient d'abord que la dialectique socratique consiste dans la recherche d'une définition réelle et non purement nominale (pp. 79-82). Ceci est en parfait accord avec les dialogues de jeunesse de Platon aussi bien qu'avec les témoignages d'Aristote et de Xénophon. Mais il soutient également que la définition socratique est une définition par le genre et la différence en s'appuyant sur l'Euthyphron (11e-12a), sur le Protagoras (329d, $349 \mathrm{~b}-\mathrm{c}$ ) et sur les Définitions ( $414 \mathrm{~d})$. La recherche socratique de la définition ne comporte rien de tel : ni le genre ni la différence. La définition par le genre et la différence appartient à la logique aristotélicienne (Topiques, I, 8, 103b15, VI, 4, 141b25, VII, 3, $153 \mathrm{bl4}$ ) et l'on pourrait en voir les premiers germes dans les dichotomies du Sophiste et du Politique. Dans le Sophiste, par exemple, Platon identifie l'eidos au genos (254a-259d), mais nous sommes loin d'une telle identification dans les dialogues de jeunesse où l'eidos est conçu, tel que l'indique d'ailleurs l'auteur, comme un universel, une essence ou encore une norme (pp. 67. 78). L'Eutbyphron (11e-12a) établit certes une distinction entre le tout et la partie en se demandant si le juste est une partie du pieux ou si c'est le pieux qui est une partie du juste. Mais on ne saurait investir cette distinction primitive du tout et de la partie de toute la richesse technique dont se trouve revêtue la distinction aristotélicienne du genre et de l'espèce. En effet, si nous nous reportons aux textes du Protagoras cités par l'auteur, nous verrons qu'il est impossible d'identifier le tout au genre aristotélicien et la partie à l'espèce. À cet endroit, il est dit que la tempérance, la justice, la sainteté sont des parties de la vertu qui est considérée comme un tout. Alors Socrate se demande si elles sont des parties au même titre que les yeux, les oreilles, le nez sont des parties du visage ou si elles sont des parties à la façon des parties d'une masse d'or (Prot. 329c-e). Il est clair par cet exemple que le Socrate du dialogue lorsqu'il parle du tout et de la partie n'a pas dans l'esprit les notions de genre et de différence spécifique. Comment peut-on, en effet, parler des yeux comme d'une espèce du visage ou encore des parties d'une même masse d'or comme d'une espèce de l'or? Le rapport envisagé ici entre la partie et le tout est un rapport de ressemblance et de dissemblance et non le rapport logique du genre et de la différence spécifique. Quant aux Définitions, elles expriment une notion de la définition de beaucoup postérieure à la recherche socratique de la définition et elles ne sauraient être une 
preuve que Socrate cherchait à définir les choses par le genre et la différence. Certes, nous n'aurions pas d'objection à ce que l'on parle, comme le fait Ross, de la distinction du tout et de la partie comme d'une anticipation de la définition par genre et espèce, mais à la condition qu'on lui conserve une certaine neutralité technique $^{3 .}$. Or cet usage technique des notions de genre et de différence spécifique fondé sur la distinction du tout et de la partie permet à l'auteur de soulever à propos de la dialectique socratique des problèmes qui lui sont tout à fait étrangers. Par exemple, le problème de savoir comment concilier la définition qui implique une composition de genre et de différence avec la simplicité de l'eidos (pp. 89-91) ou encore celui de la structure hiérarchique des Formes (pp. 91-93), problèmes, à notre avis, qui relèvent de la dialectique platonicienne et non de la dialectique socratique. Comme nous en avertit Aristote (Met. XIII, 1078b17-32), la dialectique socratique consiste dans une démarche inductive en vue d'exprimer l'universel dans une définition. Socrate cherchait l'eidos des choses, mais il ne lui arrivait pas de penser que l'eidos puisse être exprimé en termes de genre et de différence. Cette recherche appartiendra à une pensée ultérieure.

L'interprétation de la recherche socratique de la définition comme étant une recherche du genre et de la différence permet à l'auteur de passer du plan logique au plan métaphysique (p. 102). En effet, l'auteur prend le soin d'établir une différence entre la conception aristotélicienne du genre et la conception platonicienne, différence d'ailleurs à l'intérieur de laquelle on ne voit plus très bien où situer la dialectique socratique (pp. 85-89). Quoi qu'il en soit de ce problème, l'auteur soutient que le genre pour Aristote est une abstraction des espèces tandis que pour Platon il est une implication des espèces à la manière de l'implication des parties dans un tout. Par conséquent, le genre platonicien est plus riche que le genre aristotélicien, on doit lui présupposer le même degré de réalité et d'existence que ses parties. Et ceci donne au genre platonicien une densité ontologique plus riche que celle du genre aristotélicien. En se fondant sur cette conception platonicienne du genre et de l'espèce, l'auteur est amené à aborder les problèmes proprement ontologiques de la théorie des Formes séparées: la

32. RoSS, 1). Plato's Theory of Ideas, op. cit., p. 12. Voir aussi l'ouvrage de R. Robinson, Plato's Earlier Dialectic (19531. Oxford, 1962, pp. 49-60. Robinson n utilise pas les notions de genre et de différence dans son examen de la définition socratique. 
hiérarchie des Formes (pp. 91-93), l'existence et la réalité des Formes (pp. 105-106), la causalité essentielle des Formes (pp. 120-121) et la séparation des Formes (pp. 129-149). La simple énumération de ces problèmes montre par elle-même que nous sommes au coeur du platonisme des dialogues de maturité et nous ne savons pas très bien comment ils peuvent être rattachés à la dialectique socratique si ce n'est, comme s'efforce de le faire l'auteur, par une interprétation anticipative des textes de jeunesse de Platon. Par exemple, l'auteur nous dira que les textes d'Euthyphron, 11e12a, Protagoras, 329c ss., Ménon, 74-76, Lachès, 190c-d, 199e contiennent une doctrine de la hiérarchie des Formes (p. 92). C'est nous semble-t-il solliciter quelque peu les textes de jeunesse que de lire, dans la distinction du tout et de la partie identifiée au genre et à l'espèce, la doctrine d'une structure hiérarchique des Formes. En réalité, que cette doctrine soit simplement heuristique comme le soutient Cherniss ou qu'elle soit ontologique comme l'affirme R.E. Allen, elle n'est explicitée que dans le Sopbiste à travers l'exposé de la méthode de la diairesis (219a-231c) et de la communication des genres (251a-258c). En suivant l'auteur dans les méandres de son interprétation, on ne sait plus vraiment s'il est en train de nous parler de la dialectique socratique ou de la dialectique platonicienne. Son interprétation nous porte à penser que la question socratique: "Qu'est-ce que $X$ ?" ne se réduit pas seulement à la question: "Quel est l'eidos de X?", mais implique également la question à notre avis strictement platonicienne: "Quelle est la nature de l'eidos de X?" Or, pour nous, les deux questions apparaissent fort différentes. En se demandant: "Qu'est-ce que la piété? ", Socrate cherchait sans doute l'eidos de la piété, mais c'est Platon qui, reprenant l'interrogation socratique, s'est ensuite demandé quelle est la nature de l'eidos de même qu'Aristote reprenant la définition socratique lui a donné la structure logique du genre et de la différence spécifique. C'est ainsi que le statut ontologique de la dialectique socratique nous semble être construit par l'auteur sur des confusions de doctrines qui n'ont rien à voir avec la recherche socratique de la définition. Et l'on retrouve cette même confusion de doctrines en ce qui concerne l'application aux dialogues de jeunesse de Platon de la théorie référentielle de la signification (p. 113 ss.)

Nous n'avons fait qu'effleurer ici les problèmes logiques et ontologiques que pose cette interprétation de la théorie des Formes 
séparées du point de vue de l'historien de la philosophie. L'ouvrage de R.E. Allen mériterait, nous l'avouons, une critique plus détaillée que notre propos ne le permet. On ne rend pas justice à une construction aussi brillante que rigoureuse qui frappe par l'originalité et la profondeur de la pensée en quelques pages de considérations critiques. Nous sommes conscient que notre désaccord sur l'interprétation de R.E. Allen de la première théorie des Formes séparées repose finalement sur une méthode de lecture des textes anciens dont il faudrait sans doute éclaircir les principes et les présupposés philosophiques. Mais cet examen nous entraînerait sans doute trop loin. Nous aimerions seulement ajouter que l'ouvrage de R.E. Allen nous rappelle celui de G. Ryle sur Plato's Progress du point de vue de la démarche fondamentale de l'auteur ${ }^{33}$. C'est dire toute la valeur que nous lui reconnaissons pour sa force spéculative en même temps que notre profonde déception pour sa faiblesse historique.

\section{R.A. Shiner et la dernière Théorie des Formes}

Tandis que l'ouvrage de R.E. Allen soulevait des problèmes relatifs à la première théorie des Formes, celui de R.A. Shiner nous situe au coeur d'un débat contemporain sur la dernière théorie des Formes qui implique une interprétation générale des derniers dialogues de Platon. Les protagonistes de ce débat se regroupent ici autour de deux interprétations fondamentales des derniers dialogues de Platon. Les uns soutiennent que Platon n'a pas abandonné sa théorie des Formes de la maturité après les critiques du Parménide, qu'il conçoit encore les Formes comme des réalités subsistantes et indépendantes du monde sensible et que la science demeure toujours pour lui une connaissance directe des Formes in-

33. Voir notre article sur G. Ryle : Le Platon de G. Ryle, ds. Rev. Philos. Louvain, 69 (1971) 337.369. Nous signalons au lecteur les comptes rendus suivants sur l'ouvrage de R.E. Allen : A.J. Boyle. Autr. Journ. Philor. 49 (1971) 331-334, J. Dybikowski, Dialogue, 10 (1971) 565.568. D.W Hamlyn, Philosophy, 46 (1971) 170.172, G. Anagnostopoulos, G. Jonrn. Hist. Pbilos. 10(1972) 354-358, K.H. Ilting, Gnomon, 44 (1972) 326-335. R. Robinson, Mind, 81 (1972) 631-632, K.M. Sayre, Pbilos Quart., 22 (1972) 165-166. J.E. Thomas. New Scholati. 16 (1972) 275-278, I.M. Crombie, Class. Rev., 22 (1972) 330-332, R.F. Kiesau, Pbilos. Fortm, 11 (1972) 207-208, R.K. Sprague, Class. Pbilol. 67 (1972) 153-54. Sweeney, Ret. Meta., 25 (1972) 547-49, J.J. Mulhern, Journ. of Value Inquiry, 7 (1973) 71 -73. J.J. Tierney, Pbilor. Studier, vol. 21, pp. 267-268. R.E. Allen est aussi l'éditeur d'un remarquable recueil d'articles contemporains sur Platon: Studies in Plato's Metaphysics. London-New York, 1965 
telligibles ${ }^{4.4}$. Les autres affirment au contraire que Platon a pratiquement abandonné sa théorie des Formes intelligibles de la maturité ou l'a complètement révisée de sorte qu'il ne cherche plus à expliquer la science comme une connaissance directe des Formes, mais qu'il cherche une explication de la science au niveau du jugement et de la proposition ${ }^{35}$. R.A. Shiner donne le nom d'antirévisionniste à la thèse du premier groupe et celui de révisionniste à celle du second groupe. Il prend parti dans son étude pour la thèse révisionniste.

Parmi les dialogues de vieillesse de Platon, les partisans d'une interprétation antirévisionniste s'appuient surtout sur le Timée (27c-31a, 48e-52d) et le Philèbe. En effet, ces deux dialogues offrent une série de textes qui semblent à première vue nettement favoriser la présence des Formes transcendantes dans la dernière pensée de Platon et, par conséquent, de ce que R.A. Shiner appelle une ontologie dualiste et choristique caractérisée par la séparation $\mathrm{du}$ monde intelligible et transcendant et du monde sensible ( $\mathrm{p}$. 23). La démarche stratégique de l'auteur consiste d'abord à reléguer, à la suite d'Owen, le Timée parmi les dialogues de maturité

34. Parmi les représentants les plus connus du premier groupe, on nous permettra de citer : F.M. Cornford, Plato: Theory of Knouledge (1935), H.F. Cherniss, "The Pilosophical Economy of the Theory of Ideas," ds. Am. Joum. of Pbilol. 57 (1936) 445-456, D. Ross, Plato's Therry of ldeas (1951), G. Vlastos, The Third Man Argument in the Parmenides, (1954), ds. Sulties in Plato's Metaphysics, pp. 231-263, R.S. Bluck, Logos and Forms in Plato: a reply to Profesror Cross (1956), ds. Studies in Plato's Metapbysics, pp. 33.41, H.F. Cherniss, The Relation of the Timatus to Plato's Later Dialogues (1957), ds. Studies in Plato's Metaphyicr, pp. 339-378, R. Hackforth, "Platonic Forms in the Theaetetus ", ds. Clas.s. Quart. 51 (1957) 53-58. W.R. Runciman, Plato's Parmenides (1959), ds. Studies in Plato's Metaphysic, pp. 149-184, N. Gulley, Plato's Theory of Knowledge (1962), W.G. Runciman, Plato's Later Epistemology (1962), R.S. Bluck, "Knowledge by acquaintance in Plato's Theaetetus, "ds. Mind. 72 (1963) 259-263, J.M. Rist, "The Immanence and Transcendence of the Platonic Form," ds. Philologus, 108 (1964) 217-232.

35. Parmi les représentants du second groupe, nous signalons : G. Ryle, "Plato's Parmenides I, " ds. Mind 48(1939) 129.151, "Plato's Parmenides II, " ds. Mind 48 (1939) 317-321, R. Robinson, "Forms and Error in Plato's Theaetetus", ds. Philos. Rev. 59 (1950) 3-30, G.E.L. Owen. The Place of the Timaews in Plato's Dialogues, (1953). ds. Studies in Plato's Metwlyysics, pp. 313-338. R.C. Cross, "Logos and Forms in Plato," ds. Mind, 63 (1954) 133-451. D.W. Hamlyn, "The Communion of Forms and the Development of Plato's Logic, "ds. Pbilos. Quart. 5 (1955) 289-302, D.W. Hamlyn, "Forms and Knowledge in Plato's Theaetetus : a reply to Mr. Bluck ", ds. Mind, 66 (1957) 547, W. Bondeson, "Perception, true opinion and knowledge in Plato's Theaetetus, " ds. Phronesis, 14 (1969) 111.122 , M.S. Brown. "Theaetetus : knowledge as continued learning," ds. Journ. Hist. Pbilos. 7 $(1969)$ 359-379, J.M. Cooper, "Plato on sense-perception and knowledge: Theaet. 184 to 180, "ds. Ploroneris, 15 (1970) 123-146. Nous sommes conscient que cette division en deux groupes d'interprètes des derniers dialogues de Platon ne rend pas justice aux multiples nuances d'interprétation que l'on rencontre à l'intérieur de chacun de ces groupes. C'est pourquoi nous nous en sommes tenu dans notre formulation à des caractéristiques très générales. 
(p. 30) et à s'attaquer aux passages métaphysiques du Pbilèbe ${ }^{36}$. Les passages analysés sont au nombre de quatre : Philèbe, 14c-18d sur l'opposition de l'Un et du Multiple et la méthode dialectique (pp. 38-42); Philèbe, 23a-31 a sur les quatre genres suprêmes (pp. 43-48), Philèbe, 53c4-55c3 sur le plaisir-genèse (pp. 49-52), et finalement Philèbe, 55c-62a sur la hiérarchie des sciences et l'opposition entre l'Être et le Devenir (pp. 53-66). Comme on peut le constater par les intitulés, R.A. Shiner ne se donne pas la partie belle. Aussi doit-il apporter des nuances à l'interprétation révisionniste qu'il se propose de donner de ces passages métaphysiques. Il veut montrer que ces passages métaphysiques ne constituent une évidence suffisante ni pour une interprétation antirévisionniste ni pour une interprétation révisionniste. Cependant son analyse des passages se propose d'arriver à la conclusion raisonnable que le texte du Philèbe demeure cohérent avec une interprétation révisionniste des derniers dialogues de Platon, c'est-àdire qu'il peut être compris sans recourir à la théorie des Formes transcendantes de la maturité (p. 34). Mais avant d'aborder direc. tement ces passages du Philèbe, l'auteur consacre la première partie de son étude à une interprétation générale de la théorie des Formes (pp. 13-37). Il nous faut dès maintenant le suivre sur ce chemin qui nous fournira la clé de son interprétation du Philèbe.

L'auteur commence par établir une distinction qui semble à première vue anodine, mais qui va apparaître par la suite capitale dans son interprétation générale de la théorie des Formes. C'est la distinction entre le concept de $\mathrm{X}$ et les conceptions de $\mathrm{X}$. Les conceptions de $\mathrm{X}$ sont personnelles et relatives à des individus ou groupes d'individus tandis que le concept de $\mathrm{X}$ est impersonnel et $n$ 'est pas relatif à des individus ou groupes d'individus. Les conceptions de la maternité chez les Italiens ne correspondent pas nécessairement au concept de maternité. Elles peuvent être fausses à l'égard de ce concept. Cette distinction permet à l'auteur de rejeter l'interprétation ontologique de la théorie des Formes dans les dialogues de jeunesse telle que proposée par R.E. Allen dans l'ouvrage

36. Ce procédé de renvoi chronologique du Timée aux dialogues de maturité illustre ce que nous disions plus haut sur le présupposé philosophique des analystes d'Oxford. Ce dialogue est de fait encombrant pour tout interprète qui soutient l'abandon de la théorie des Formes transcendantes dans les derniers dialogues. 
que nous venons d'analyser ${ }^{37}$. Pour Allen, en effet, la théorie socratique des Formes implique une ontologie parce que l'eidos est la nature même des choses, leur réalité et exprime un phénomène objectif et non pas subjectif. Pour R.A. Shiner, la théorie socratique des Formes n'implique aucun fondement ontologique, mais seulement la distinction épistémologique entre le concept de $\mathrm{X}$ et les conceptions de X. Par exemple, dans le Lachès, Socrate discute de diverses conceptions du courage en vue d'établir le concept de courage (p. 20). D'ailleurs, nous avertit R.A. Shiner, il faudrait distinguer entre deux groupes de dialogues de jeunesse: d'une part, le Charmide, le Lachès et le Lysis, d'autre part, l'Euthyphron, l'Hippias Majeur et le Ménon. Ces derniers seulement utilisent un vocabulaire plus technique qui laisse entrevoir une anticipation de la théorie des Formes, par exemple l'usage d'expressions telles que aviò tó (Euthy. 6d10, Hipp. Maj. 288a9,

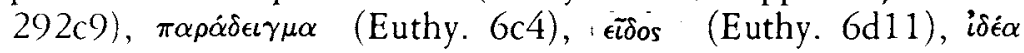
(Euthy. 6d11, Mén. 72c7). Cependant on ne saurait conclure de ces expressions à une implication ontologique en ayant recours comme le fait Allen à la causalité des Formes qui est un élément de la théorie des Formes Transcendantes (pp. 27-28). Bref, si nous comprenons bien la position de R.A. Shiner, l'eidos dans la dialectique socratique est un concept sans fondement ontologique (p. 29). Ce fondement ontologique n'apparaît que dans la théorie des Formes Transcendantes de la maturité avec la thèse de la séparation du monde sensible et du monde intelligible. C'est ce que R.A. Shiner appelle le dualisme ontologique et choristique de la maturité de Platon. Dès lors, la théorie des Formes Transcendantes doit être comprise comme une interprétation philosophique particulière de la nature des concepts (p. 22). Elle implique non seulement la distinction entre le concept et les conceptions, mais elle va plus loin que la dialectique socratique en affirmant que les concepts existent indépendamment de l'esprit humain (p. 23). Or, dans les dialogues critiques, Platon abandonne cette ontologie dualiste et choristique, c'est-à-dire la séparation du monde sensible et du monde intelligible. Le Parménide donne le coup de grâce à cette ontologie dualiste et si l'on réfere l'auteur à Parménide, 135 b5-c3 où il est dit après la critique des Formes transcendantes qu'on doit pourtant les maintenir au risque de détruire la possibilité de toute dialec-

37. ALLEN, R.E., Plato's Eutbyphro and the Earlier Theory of Forms, op. cit., pp. 105.128. 
tique, R.A. Shiner répond que la nécessité de maintenir les Formes signifie seulement le maintien de la distinction entre le concept et les conceptions de $\mathrm{X}$, doublée de la nécessité de poser le concept intemporel et indépendant de l'esprit humain (p. 32). On ne retrouve donc plus dans le Parménide, le Théétète et le Sophiste la thèse de la séparation des deux mondes et par conséquent l'ontologie dualiste de la théorie des Formes Transcendantes.

Arrêtons-nous un moment sur cette interprétation générale de la théorie des Formes. Nous serions fondamentalement d'accord avec la critique qu'adresse R.A. Shiner à l'interprétation par R.E. Allen de la dialectique socratique. D'une part, il faut distinguer entre la théorie des Formes transcendantes de la maturité et la théorie des Formes de la jeunesse. L'interprétation de R.E. Allen obscurcit cette distinction en parlant de la dialectique socratique comme d'une théorie des Formes séparées et en interprétant la séparation comme étant celle du concept et des cas particuliers. On

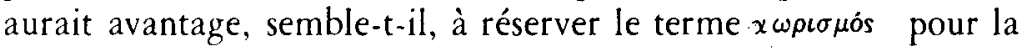
séparation du monde sensible et du monde intelligible. D'autre par, R.A. Shiner a raison de refuser la causalité essentielle des Formes dans la dialectique socratique. Celles-ci jouent sans doute un rôle d'ordre cognitif dans la démarche socratique, mais il faut attendre la dialectique platonicienne pour leur voir assigner un rôle proprement ontologique. Cependant, notre critique de R.E. Allen irait encore plus loin que celle de R.A. Shiner. Pour nous, il n'y a pas de théorie socratique des Formes et l'historien de la pensée grecque aurait intérêt à bannir cette expression de son vocabulaire pour éviter toute confusion avec les doctrines platoniciennes. La dialectique socratique est une recherche de l'eidos de $\mathrm{X}$ et Socrate ne semble pas avoir été préoccupé de construire une théorie de l'eidos qui comporterait, comme c'est le cas pour Platon, des conséquences ontologiques, épistémologiques, éthiques et politiques. On ne trouve rien de tel dans la méthode socratique de la définition. Quant à la théorie des Formes Transcendantes nous ne croyons pas qu'il faille la considérer comme une interprétation philosophique de la nature des concepts. Ce serait réduire une théorie ontologique à une théorie épistémologique comme semble le laisser croire le point (iv) du tableau que nous donne R.A. Shiner en page 23. Les Formes platoniciennes ne sauraient être comprises comme des concepts indépendants de l'esprit et rien n'indique dans le texte de Parménide, 132b3-c8 que le terme vónua doit être pris au sens de 
" conception" pour faciliter cette interprétation des Formes. Lorsque le Parménide affirme que l'eidos n'est pas un noéma, il faut comprendre que l'eidos n'est ni une pensée, ni un concept, ni une conception, ni quoi que ce soit qui puisse se produire dans les âmes (Parm. 132b4-6). Car s'il en était ainsi, poursuit le Parménide, on se verrait réduit à l'alternative suivante : ou tout serait fait de pensées et tout penserait ou tout serait pensées mais privé de penser. Il faut donc éviter d'identifier eidos et noéma, le premier désignant la réalité et le second la pensée. Par conséquent, la théorie platonicienne des Formes n'est pas une théorie sur la nature du concept, mais une théorie sur la nature du réel. Ainsi lorsque Platon affirme dans le Parménide (135b-c), après sa critique de la théorie des Formes Transcendantes, que la négation de celles-ci entraînerait l'abolition de la puissance dialectique, il ne fait certainement pas appel à la distinction entre le concept de $\mathrm{X}$ et les conceptions de X pour répondre aux critiques que l'on vient de formuler (p. 32). Au contraire, la transcendance des Formes doit être maintenue, mais on doit s'entraîner davantage dans les exercices dialectiques en vue de résoudre ces difficultés (Parm. 135d-e). Et la seconde partie du Parménide est un exemple de ce genre d'exercices dialectiques. Ce passage à lui seul constitue tout un programme pour les derniers dialogues de Platon et pour leur interprétation.

Dans la seconde partie de son étude, R.A. Shiner nous donne, sur le fond de cette interprétation générale de la théorie des Formes, sa lecture révisionniste des quatre passages métaphysiques du Philèbe et cherche à montrer qu'une telle lecture demeure aussi cohérente avec le texte platonicien qu'une lecture antirévisionniste. Nous ne pouvons ici suivre l'auteur dans tous les détails d'une analyse méticuleuse et rigoureuse de tous ces textes. Qu'il nous suffise d'en indiquer les conclusions les plus pertinentes. Le Philèbe, 14c-18c sur le problème de l'Un et du Multiple présente des affinités terminologiques indubitables avec la théorie des Formes transcendantes (p. 40). Mais les questions métaphysiques soulevées dans ce passage demeurent sans réponse parce que la théorie des Formes Transcendantes demeure inadéquate pour les résoudre. Certes cela ne prouve pas que Platon ait abandonné l'ontologie choristique de la théorie des Formes, mais qu'il est possible de lire le passage sans faire intervenir celle-ci (pp. 41-42). Il en est de même pour le passage de Philèbe (23c-31a) sur les genres su- 
prêmes où R.A. Shiner se rallie, contre Cherniss, à l'interprétation d'Owen selon laquelle la continuité affirmée par le texte entre le

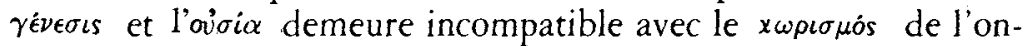
tologie de la maturité (p. 44), de sorte que ce passage peut être compris sans référence à la Transcendance des Formes (p. 67). Dans le passage de Philèbe, 53c-55a qui est de nature éthique plus qu'ontologique puisqu'il traite de la genèse du plaisir, les termes

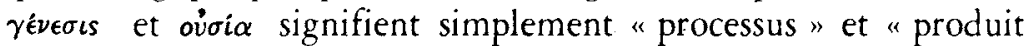

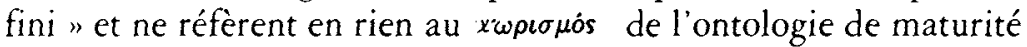
(p. 67) comme le soutient Cherniss contre Owen. Enfin, le dernier passage examiné (Phil., 55c-62a), qui, du point de vue de la terminologie et de la doctrine, rappelle le plus la théorie des Formes Transcendantes (p. 54), est également compatible avec une lecture révisionniste. En effet, l'auteur montre, textes à l'appui, que Platon exprime dans ce passage une conception de la science incompatible avec celle de la maturité (p. 60) aussi bien qu'une conception de la réalité qui consiste à remplacer la transcendance des Formes par l'existence de concepts intemporels et immuables (p. 63). Certes, nous avertit l'auteur (p. 65), sa lecture révisionniste ne cherche pas à prouver positivement que Platon a abandonné sa théorie des Formes transcendantes, il concède même que l'on puisse faire de ces textes une lecture antirévisionniste, mais il veut simplement montrer la possibilité de lire tous ces textes sans présupposer les Formes Transcendantes.

Ces conclusions trop brièvement résumées, nous l'avouons, au risque de ne pas rendre justice aux pénétrantes analyses de l'auteur, nous amènent à formuler deux remarques finales avant de quitter cet ouvrage. La première est que la lecture ou l'interprétation révisionniste de la dernière théorie des Formes n'est pas, chez R.A. Shiner, aussi radicale que celle de R. Robinson. En effet, l'auteur ne va pas jusqu'à vouloir prouver que Platon a abandonné sa théorie des Formes Transcendantes dans sa dernière philosophie ni que l'explication de la science doit être cherchée dans l'analyse du jugement et de la proposition. Le révisionnisme de R.A. Shiner nous apparaît davantage comme un révisionnisme mitigé. L'auteur soutient, d'une part, qu'il est possible de lire les passages métaphysiques du Philèbe sans faire appel à la Transcendance des Formes et, d'autre part, que la nouvelle conception de la science dans le Philèbe consiste seulement à placer à côté de la dialectique considérée dans la République comme seule science, d'autres 
savoirs qui dans le Pbilèbe reçoivent le nom de sciences (pp. 5657). Le révisionnisme de R.A. Shiner repose en définitive sur le refus d'accepter dans sa lecture du Philèbe la présence d'une ontologie de type transcendant. Sur ce point précis, il se meut dans $k$. courant des interprétations analytiques de la dernière philosopli. de Platon. Mais du coup son interprétation qui cherche à montrer des modifications majeures dans la conception de la connaissance et de la réalité chez le dernier Platon le rapproche, bien malgré lui, d'une interprétation antirévisionniste. En effet, peu d'interprètes antirévisionnistes refuseraient d'admettre, tout en maintenant la présence de la théorie des Formes Transcendantes dans les derniers dialogues, que celle-ci a subi, après les critiques du Parménide, de profondes modifications du point de vue ontologique et épistémologique. La question est de savoir comment comprendre et expliquer ces modifications. En réalité, et c'est là notre seconde remarque, un interprète antirévisionniste pourrait reprendre la même démarche que celle de R.A. Shiner, mais en sens inverse. Au lieu de mettre en relief les différences d'ordre terminologique ou doctrinal entre le Pbilèbe et la République (pp. 55-58), on pourrait aussi facilement mettre en relief leurs affinités. Par exemple, l'usage du terme єкєі̃ $\alpha$ en Philèbe, 59c3, qui est un terme technique pour désigner les Formes Transcendantes ${ }^{38}$ et que l'auteur ne mentionne pas, aussi bien d'ailleurs que les oppositions mentionnées en p. 53 entre l'être et le devenir, l'opinion et la science, la connaissance de ce qui change et celle de ce qui ne change pas, oppositions très courantes dans la philosophie de la maturité. Inutile d'insister sur tous ces textes que l'auteur connaît fort bien. Mais si ces deux démarches à sens inverse sont possibles et qu'elles entraînent deux interprétations différentes de la dernière ontologie de Platon, la question à poser demeure celle de savoir qui a un intérêt théorique à supprimer l'ontologie transcendante admise par ailleurs par une forte tradition des platonisants anciens et modernes? Cette question nous renvoie non pas au texte platonicien, mais bien plutôt à la philosophie de l'interprète. Dès lors que devient le platonisme sans son fondement ontologique des Formes Transcendantes? Peut-être sans doute quelque chose d'analogue au kantisme sans son fondement épistémologique de la théorie des jugements a

38. Phéd. 7445, d6, 75b6, Banq. 212 al, Rép. VI, 510a3, Phèdr. 250al, Parm, 133e5.6, 134al, 134d5. Pol. 300e2, d7, 30lal, Tim. 69a3 
priori". C'est tout simplement un platonisme qui boîte.

\section{Le Pbédon de D. Gallop}

C'est dans la plus pure tradition des analystes d'Oxford que D. Gallop nous présente sa traduction anglaise et son commentaire du Phédon de Platon. L'ouvrage fait partie de la collection "Clarendon Plato Series" publiée à Oxford, à côté du Théétète de J. McDowell (1973), du Philèbe de J.C.B. Gosling (1975) et du Protagoras de C.C.W. Taylor (1976). L'intérêt de cette collection pour un platonisant est de lui fournir une lecture analytique de l'ensemble des dialogues de Platon si le projet est mené à terme. Nous laissons ici à nos collègues anglophones le soin d'apprécier à sa juste valeur la traduction que nous donne D. Gallop du texte grec du Phédon. Nous ferions seulement remarquer que l'auteur a attaché une grande importance à la traduction des termes techniques qu'il prend d'ailleurs toujours soin de justifier soit dans ses commentaires soit dans des notes particulières disponibles à la fin de l'ouvrage (pp. 226-238). Le commentaire lui-même suit de près le texte platonicien qu'il soumet à une analyse rigoureuse du langage où se révèlent à la fois l'acribie de l'interprète et la force conceptuelle de ses interventions sur le texte platonicien. Il nous apparaît difficile dans le peu d'espace dont nous disposons d'entrer ici dans lés détails techniques d'un commentaire qui, avouons-le, ne se laisse pas facilement prendre en défaut. Nous aimerions plutôt nous en tenir à deux considérations générales sur cette lecture analytique du texte platonicien, considérations que nous ferons du point de vue d'un lecteur de Platon qui appartient à ce qu'on a appelé la tradition continentale.

Ce qui nous a frappé d'abord dans les commentaires de $\mathrm{D}$. Gallop, c'est la quasi-absence de référence à l'horizon historique dans lequel se situe normalement le discours platonicien du Phédon, c'est-à-dire comme texte philosophique du IVe siècle avant notre ère ${ }^{i 0}$. Certes l'auteur ne manque pas de signaler quelques données historiques (p. 74, 78 etc.) ou encore de relier le

39. Nous ne connaissons malheureusement qu'un seul compte rendu de l'ouvrage, de C.J. Mc Knight, Pbilos. Books, 16, n. 2 (1975) 29-30.

40. Comme point de comparaison, nous invitons le lecteur à comparer le commentaire de D Gallop et celui, par exemple, de Gauthier-Jolif sur l'Éthique à Nicomaque d'Aristote (19581959) pour comprendre ce que nous entendons par l'horizon historique d'un texte. 
texte platonicien à d'autres textes qui lui sont contemporains ( $\mathrm{p}$. 140, 148 etc.), mais il faut avouer que ce procédé d'analyse du texte ne fait pas partie de sa méthode de travail. Pourtant comme oeuvre située dans le temps, le Phébon se fait l'écho des croyances religieuses de l'époque, celles partagées par la conscience populaire ou encore développées dans les cercles plus fermés de l'orphisme et du pythagorisme, il emprunte ses concepts à la pensée présocratique et même à la tradition homérique, il dialogue avec la sophistique de l'époque, bref, le discours platonicien du Phédon s'enracine dans un monde culturel différent du nôtre. Il nous semble qu'une bonne partie de l'intelligibilité du texte repose sur la capacité du lecteur à le relier au contexte culturel de l'époque pour en montrer l'originalité et la pertinence eu égard aux problèmes que se posait l'homme grec de cette époque. Comment peut-on comprendre le Phédon sans faire référence à toutes ces coordonnées historiques? La lecture analytique du Phédon abolit ce contexte historique du discours platonicien pour demander uniquement à l'analyse conceptuelle la clé d'explication du texte platonicien. D. Gallop accepte ce pari, mais nous devons reconnaître qu'il est gros de conséquences, et surtout pour un auteur comme Platon dont le discours philosophique est rempli d'éléments qui appartiennent à la culture de son époque et qui ne prennent souvent tout leur sens que par rapport à celle-ci. L'effet le plus visible, nous semble-t-il, de cette absence d'horizon historique est une transformation presque imperceptible au cours du commentaire du discours platonicien en un véritable discours contemporain. Nous disons imperceptible parce que le commentateur s'appuie toujours sur le texte, ce qui donne au lecteur l'impression de travailler sur la pensée de Platon alors qu'en réalité il travaille davantage sur celle de l'interprète de Platon.

Prenons pour exemple les longs commentaires que nous donne $\mathrm{D}$. Gallop sur le problème du suicide au début du dialogue (pp. 76-85), commentaires qui comprennent un tableau de formules logiques en p. 83. Le commentateur nous parle d'un paradoxe qui consiste pour un authentique philosophe, d'une part, à désirer la mort qu'il considère comme un bien, et d'autre part, à refuser de tendre vers ce bien en se donnant lui-même la mort. Le commentateur refuse de considérer que le paradoxe est résolu en alléguant, comme le fait Socrate, que l'homme est la propriété des dieux ( $\mathrm{p}$. 76). Or, en réalité, le paradoxe est résolu dans un contexte de 
croyance religieuse que Cébès accepte d'emblée (62c). L'intention de Platon n'est pas ici de traiter le problème du suicide d'une façon dialectique, auquel cas on serait autorisé à lui appliquer le procédé de l'analyse conceptuelle, mais de résoudre une objection de Cébès en faisant appel à une croyance religieuse dont la formule se trouvait dans les Mystères (62b). C'est par l'abolition du contexte religieux du discours platonicien que l'on crée le paradoxe sur lequel aura prise l'analyse conceptuelle et que le suicide devient un véritable problème logique. Â partir de ce moment, le commentateur se trouve aux prises avec des difficultés qui sont étrangères au texte platonicien et qui relèvent du discours contemporain, c'est-à-dire de son propre discours conceptuel. En effet, le contexte religieux du texte platonicien résout le paradoxe du suicide du point de vue de la conscience religieuse de l'époque et non du point de vue de la raison logique. Ce dernier point de vue demeure étranger au texte platonicien. Dès lors, ne serait-il pas plus fécond pour l'intelligibilité du texte platonicien de se demander comment la conscience religieuse de l'époque considérait le suicide et comment se situe le texte de Platon par rapport à celle-ci ? Nous ne croyons pas qu'on puisse faire l'économie de l'histoire à si bons frais dans l'étude des textes philosophiques au nom de la pure spéculation logique.

Ce qui nous frappe aussi dans les commentaires du Pbédon de D. Gallop, c'est l'abolition du dialogue comme véhicule de la philosophie de Platon. La lecture analytique du Phédon procède comme si le dialogue platonicien était un exposé uniforme, progressif et logiquement articulé de la pensée de Platon à la manière d'un texte de Descartes ou de Kant. Cette conception du texte platonicien va à l'encontre des études d'éminents platonisants tels que Hirzel, Friedländer, Schaerer, Goldschmidt parmi tant d'autres et qui nous ont apporté une perception toute nouvelle du dialogue comme genre littéraire ${ }^{41}$. Le dialogue platonicien est à la fois une oeuvre d'art et une oeuvre philosophique ; comme genre littéraire, il emprunte ses éléments à l'épopée, à la comédie et à la tragédie, à l'histoire et à la procédure judiciaire de la question et de la

41. Sur le dialogue platonicien comme genre littéraire on pourra consulter : R. Hirzel, Der Dialog (1895), Hildesheim G. Olms, 1963, P. Friedländet, Platon (1928), Berlin, 1964, vol. I, R. Schaterer. La Question Platonicienne (1938), Paris, 1969, V. Goldschmidt, Les Dialosues de Plutm (1947). Paris, 1963, K. Gaiser, Protreptik und Paranese hei Platon. Untersuchungen zur Form des platonischen Dialogs, Stuttgart, 1959, H. Gundert, Dialog und Dialektik. Zur Struktur des platonischen Dialogs, Amsterdam, 1971. La citation de R. Schaerer est tirée de son ouvrage cité plus haut, en page 13 de l'édition de 1969 
réponse ; bref, le dialogue platonicien n'est pas un traité de philosophie, mais bien plutôt un véritable drame philosophique. C'est pourquoi R. Schaerer ne craignait pas d'écrire: "Chez Platon, l'élément premier, c'est le dialogue et l'élément secondaire, l'idée, ou, si l'on veut, l'idée n'a de signification que par sa place dans le dialogue; elle n'en constitue ni la raison d'être ni le terme." Cette conception du dialogue platonicien se situe à l'opposé de la conception sous-jacente à la lecture analytique du texte où c'est le concept ou l'idée, pour employer le langage de Schaerer, qui retient constamment l'attention du commentateur sans que celui-ci arrive à se soucier de la manière dont le concept surgit et se développe dans la discussion dialoguée. La lecture analytique abolit ainsi le dialogue pour lui substituer un texte purement linéaire. Le dialogue platonicien n'est pas un jeu de concepts, mais un drame qui met aux prises des interlocuteurs qui évolueront sous l'influence de la discussion dialoguée. Comme nous le rappelle le Phèdre, le dialogue platonicien est une véritable psychagogie (271c-d), une façon de mener les âmes qui tient toujours compte de l'état d'esprit des interlocuteurs. Le dialogue platonicien, à l'instar du drame antique, n'articule pas des concepts, mais les étapes d'un cheminement des interlocuteurs du drame qui comporte des niveaux de langage qu'une simple lecture analytique ne permet pas de percevoir.

Le Phédon constitue un bel exemple de ce cheminement progressif des interlocuteurs qui aboutit finalement à une véritable démarche dialectique dans l'argument final sur l'immortalité de l'âme. Tout ce qui précède constitue une préparation ces interlocuteurs à comprendre et à accepter cette démarche dialectique finale. Par conséquent, ce serait fausser notre compréhension du Phédon que de le considérer comme un bloc uniforme d'une démarche logique possédant du commencement à la fin le même type de rationalité. En réalité, les trois premiers arguments du Pbédon appartiennent à la thétorique philosophique et ne dépassent pas le niveau de la simple croyance. Le but de ces discussions préliminaires est de créer chez les interlocuteurs une certaine sérénité face à la mort, de les débarrasser de leur peur pour les rendre aptes à comprendre l'argument dialectique. Il ne s'agit pas ici d'arguments logiques, mais plutôt d'une véritable protreptique dont la valeur ne saurait être jugée à la lumière de l'analyse conceptuelle, mais du point de vue de son efficacité sur l'esprit des interlocuteurs evi 
égard à ce qu'ils sont et à ce qu'ils croient. Cette protreptique n'est pas centrée sur les concepts, mais sur les personnages du drame. C'est ainsi que Socrate décrit son attitude sereine devant la mort (60b-69e) en la présentant à des interlocuteurs, amis de la philosophie, comme une véritable libération de la pensée. Il essaie de dissiper leur crainte chimérique de la mort fondée sur leur croyance homérique en l'âme-souffle en leur rappelant deux théories auxquelles sont attachés ses interlocuteurs : la théorie des contraires et celle de la réminiscence (70c-78b), et cela non pas parce qu'ils considèrent ces arguments comme logiquement irréfutables, mais uniquement en vertu de leur valeur protreptique. Il leur fait ensuite une longue exhortation à la vie philosophique comme étant la meilleure préparation à la mort (82c-84c), précédée d'un mythe sur la destinée de l'âme $(80 \mathrm{e}-82 \mathrm{c})^{42}$. Toute cette protreptique qui vise un changement d'attitude des interlocuteurs du drame ne se fonde pas sur la raison logique, mais sur d'antiques traditions religieuses $(60 \mathrm{~d} 2,61 \mathrm{~b} 3,62 \mathrm{~b} 2-5,63 \mathrm{c} 6$, etc.), sur les dires des poètes $(60 \mathrm{dl}, 61 \mathrm{~b} 6,70 \mathrm{a} 2-4$, etc.) et sur des mythes $(80 \mathrm{e}-82 \mathrm{c})$. Elle ne vise pas à la certitude logique, mais uniquement à la croyance et à l'espoir (61e1-2, 61b4-5, 63c5, 66b2, 67b7, 67c1, 69d1). La nature propre du discours protreptique échappe complètement à la lecture analytique en lui faisant subir le traitement d'un véritable discours logique, opération qui nous semble analogue à celle qui consisterait à faire subir à un poème de Byron le traitement de l'analyse conceptuelle. Cette opération a-t-elle un sens? Oui, sans doute, pour le logicien, mais non pas pour l'intelligibilité même du poème. C'est ainsi qu'une lecture analytique d'un dialogue platonicien, bien qu'elle puisse être un excellent exercice de l'esprit, risque de le fermer à l'intelligibilité propre du platonisme dans son contenu historique et dans sa valeur psychagogique. Le type de rationalité que développe la lecture analytique du dialogue platonicien ne coïncide pas dans son uniformité logique avec le type de rationalité multiforme de la discussion dialoguée. C'est pourquoi la logique spécifique du texte se trouve ainsi trahie par la logique propre du commentateur.

42. Sur la valeur protreptique du Phédon, voir l'excellent ouvrage de A.J. Festugière, Les Trois Protreptiquer de Platon, Paris, Vrin, 1973 que nous avons recensé dans la revue Dialogue 13 (1074) 807-810. 


\section{Le Sophiste de P. Seligman}

Le Sophiste est, avec le Théétète, l'un des dialogues de Platon qui a suscité le plus d'intérêt chez les platonisants contemporains. Cette ferveur récente peut facilement se comprendre si l'on considère que les problèmes soulevés dans ces deux dialogues rejoignent les intérêts épistémologiques, logiques et linguistiques de la philosophie contemporaine. Mais du coup ces intérêts nous aident à comprendre pourquoi les nouvelles interprétations du Sophiste ont tendance à accentuer l'aspect logique du dialogue au détriment de son aspect ontologique, voire même à réduire des problèmes ontologiques à des problèmes logiques. Cette tendance, à vrai dire, était déjà présente, mais d'une façon beaucoup plus nuancée, chez des platonisants du début du siècle tels que H. Jackson (1885), L. Campbell (1894) et A.E. Taylor (1926) qui soulignaient l'aspect logique du Sophiste sans pourtant aller jusqu'à nier son aspect ontologique $e^{+3}$. Les platonisants plus récents ont poussé cette tendance à l'extrême. Pour Hamlyn, par exemple, la plus grande partie du Sophiste traite de questions logiques et particulièrement de logique des propositions ${ }^{44}$. Selon Peck, les discussions du Sophiste portent essentiellement sur le problème sophistique de la prédication, de l'usage des termes "être " et " n'être pas " dans la proposition et n'ont rien à voir avec la notion ontologique de participation telle que soulevée dans la première partie du Parménide. ${ }^{45}$ Xenakis soutient pour sa part que les genres du Sophiste ne sont pas des Formes au sens classique du terme, mais des concepts dont la combinaison constitue la proposition prédicative et que la dialectique est tout simplement un art de combiner des concepts comme la grammaire combine des lettres et la musique des sons ${ }^{46}$. Des positions analogues sont adoptées par Trevaskis ${ }^{47}$. Ainsi toute une tendance récente chez les platonisants consiste à faire une lecture logique et linguistique du Sophiste qui supprime, à toutes fins pratiques, la perspective ontologique du dialogue.

43. Voir à ce sujet : H. Jackson, "Plato's Later Theory of Ideas, V, The Sophist, " ds. Journ. Philol. 14 (1885) 225-226, L. Campbell, B. Jowett, Plato's Republic, (1894), Oxford, Clarendon Press, vol. II, p. 61, A.E. Taylor, Plato, London, Methuen, 1926, p. 387

44. HAMLYN, D.W. "The Communion of Forms and the Development of Plato's Logic, "ds. Pbilos. Quart. 5 (1955) 289-302.

45. PECK, A.L.. "Plato and the megista gene of the Sophist ". A reinterpretation, ds. Class. Quart. $46(1952) 32-56$

46. XENAKIS, J., "Plato's Sophist : a defense of negative expressions and a doctrine of sense and truth, "ds. Pbronesis 4 (1959) 29-43.

47. TREVASKIS, J.R., "The megista gene and the Vowell Analogy of Plato, Sophist 253, "ds. Phronesis 11 (1966) 99-116. 
Le Sophiste de P. Seligman va à l'encontre de cette tendance en adoptant la perspective ontologique sans nier cependant les apports logiques du dialogue (pp. 1-4). Sur ce point précis sa perspective fondamentale rejoint celle de Diès, de Cornford, de Runciman et de tant d'autres platonisants ${ }^{48}$. Il nous en explique luimême les raisons en pp. 96-97. D'abord, la négation de la possibilité du jugement faux s'enracine dans l'ontologie parménidienne et il apparaît raisonnable que le problème soit résolu à ce niveau ontologique. Ensuite pour Platon la signification (meaning) ne dépend pas seulement de la nature du discours ni de la structure des éléments dans la proposition mais aussi de la structure de la réalité que le discours exprime et qu'il reflète. Enfin, le problème du jugement faux conçu comme le fait de dire ce qui n'est pas engage fondamentalement Platon dans le problème ontologique de l'être et du non-être. Ainsi selon Platon, le jugement faux participe à la fois de l'être et du non-être dans la mesure où il est réellement et véritablement un jugement faux (263d4). La façon la plus courante d'abolir cette perspective ontologique du Sophiste est de ramener les termes " être " et " n'être pas " à un usage strictement linguistique ou encore à les considérer comme de purs connecteurs. Mais dans ce cas, quel sens peut-on donner à l'exposé central du Sophiste sur les théories pluralistes et unitaires de l'être (242b245e), sur les théories matérialistes et idéalistes (245e-247d) qui soulèvent les problèmes fondamentaux de la pensée présocratique? Comment ces discussions peuvent-elles être pertinentes si elles ne portent pas sur des conceptions diverses de la réalité, mais simplement sur un usage linguistique du verbe "être "? Nous serions donc porté ici à donner raison à $\mathrm{P}$. Seligman lorsqu'il adopte la perspective ontologique dans sa lecture du Sophiste. Pour nous, les discussions du Sophiste se pousuivent à trois niveaux : le niveau ontologique de la communication des Formes-Genres, le niveau épistémologique et logique de la vérité et de la fausseté du jugement et le niveau sémiotique de la proposition. Ces trois niveaux demeurent intimement liés entre eux de sorte que la négation de l'un empêche la compréhension de l'autre.

Ce qui permet à $\mathrm{P}$. Seligman de maintenir cette perspective

48. DIES, A., La définition de l'Être et la nature des Idées dans le Sophiste de Platon (1909), Paris, Vrin, 1963, CORNFORD, F.M., Plato's Theory of Knowledge (1935), London, Routledge and Kegan Paul, 1964, RUNCIMAN, W.G., Plato's Later Epistemology, Cambridge. In. Press, 1962. 
ontologique dans sa lecture du Sophiste, c'est le souci constant qu'il manifeste de situer la problématique générale du dialogue dans son contexte historique (pp. 5-11, 15, 16, 20-21, 25, 26, 44, 56, 64, etc.). Cette méthode de travail permet au commentateur de comprendre la pensée de Platon dans le Sophiste par rapport à celle de Parménide et des sophistes et lui évite d'injecter dans le texte platonicien des considérations qui lui sont étrangères. En réalité, la problématique générale du Sophiste s'enracine, d'une part, dans l'héritage de Parménide, et dans celui de la sophistique, d'autre part. L'auteur montre comment la dichotomie radicale de l'être et du non-être chez Parménide pouvait conduire à la thèse sophistique de l'impossibilité du jugement faux (Soph. 260c) et comment Platon cherchera dans le Sopbiste à renverser la thèse sophistique en commettant le parricide contre Parménide par l'établissement d'un être du non-être (241d-e). C'est ainsi que chaque section du Sophiste sera analysée en tenant compte à la fois du texte et du contexte historique et en insérant au cours de l'analyse des discussion sur certaines interprétations les plus pertinentes des dernières années.

À défaut d'entrer dans les détails de cette longue analyse, nous aimerions signaler au lecteur le passage, à notre avis, le plus original de l'ouvrage qui expose l'interprétation de la symploke eidon du $259 \mathrm{e}$ et que nous avouons n'avoir encore rencontrée nulle part au cours de nos lectures sur le Sophiste (pp. 89-97). L'auteur commence par distinguer trois types d'interprétations du symploke eidon. Le premier comprend la symploke eidon comme une combinaison d'un sujet et d'un prédicat à l'intérieur de la proposition, ce qui répondrait au problème soulevé en $251 \mathrm{a}$-b de l'impossibilité de la prédication. Mais cette interprétation semble trop étroite pour l'auteur (p. 90) parce qu'elle repose finalement sur une compréhension purement logique du dialogue et qu'elle ne rend pas compte de 260a 1-6, où il est dit que le discours est l'un des genres de l'être. Le second type d'interprétation se situe dans la perspective ontologique et comprend la symploke eidon comme une combinaison mutuelle de Formes-Genres. Dans tout jugement ou proposition, nous combinons des Formes et nous pouvons faire cela parce que justement les Formes-Genres se prêtent à de telles combinaisons. C'est l'interprétation bien connue de Cornford (1935), Bluck (1957) et Gulley (1962). Mais cette interprétation présentait également une difficulté. Lorsqu'on parle de combinaison des 
Formes, il nous faut au moins deux Formes. Or, dans les exemples donnés par Platon, "Théétète est assis " ou "Théétète vole ", on ne trouvait qu'une Forme combinée à un sujet empirique. On pouvait sans doute, à la suite de Cornford, résoudre la difficulté en supposant que Théétète participe à la Forme homme et que les exemples révèlent une combinaison entre la Forme homme et la Forme "être assis". Mais on devait bien avouer que cette interprétation n'était pas explicite dans le texte. P. Seligman propose, pour sa part, de pousser encore plus loin l'aspect ontologique du passage et de comprendre la symploke eidon en rapport avec 240 c 1 , où il est dit que l'être s'enlace (symploke) a non-être. Par conséquent puisque le discours appartient au genre de l'être (260a 1-6) et que l'être se combine toujours au non-être (240c1), on peut comprendre qu'en 259 e la symploke eidon ne se réfère pas au contenu du discours ou du logos, c'est-à-dire à des Formes déterminées telles que " homme ", "être assis ", mais simplement à la détermination formelle du logos, c'est-à-dire à la combinaison de l'être et du non-être. Du coup, on peut expliquer la possibilité du jugement faux par cette combinaison ontologique d'être et de nonêtre dans le discours aussi bien que dans la réalité. Cette interprétation, à notre avis, mériterait qu'on y prête attention et devrait être examinée dans tous ses détails. Malheureusement, nous devons quitter l'ouvrage de P. Seligman pour aborder brièvement le Timée de L. Brisson ${ }^{49}$.

\section{Le Timée de L. Brisson}

L'ouvrage de L. Brisson se présente comme un commentaire systématique du Timée de Platon. Dans la tradition française des platonisants, nous ne possédions jusqu'ici que les volumineuses Études sur le Timée de Platon de Th. H. Martin qui dataient déjà de 1841 et dont les deux tomes viennent de recevoir l'honneur d'une réimpression à New York chez Arno Press dans la collection " History of Ideas in Ancient Greece ", dirigée par l'éminent platonisant Gregory Vlastos (1976). L'ampleur des recherches effectuées au cours de ce commentaire autour du Timée de Platon qui embrasse pour ainsi dire toute la tradition platonicienne, aussi bien que la rigueur et la profondeur déployés dans la compréhension

49. On trouvera un compte rendu de l'ouvrage par J. Rudoff, Rev. of Met, 28 (1974-1975) 567.8 . 
philosophique du texte platonicien pourraient bien faire de ce commentaire du Timée l'ouvrage à consulter pour les platonisants de cette fin du XXe siècle. Nous avons déjà consacré un long compte rendu à cet ouvrage dans la revue Dialogue, 15 (1976) 162.169; il nous semblerait fastidieux d'y revenir. Qu'on nous permette donc ici de rappeler brièvement le contenu de l'ouvrage et de formuler deux brèves remarques critiques.

Le titre de l'ouvrage : le Même et l'Autre dans la structure ontologique du Timée de Platon, nous servira d'amorce à une articulation générale du contenu. D'abord, le terme structure, comme nous en avertit l'auteur (pp. 17-18), ne doit pas être compris dans un sens «structuraliste ", mais " néo-positiviste ». On doit donner à ce terme une signification philosophique minimale, celle d'un " tout formé d'éléments solidaires tels que chacun dépend des autres et ne peut être ce qu'il est que dans et par sa relation avec eux ". Cette structure est dite ontologique parce qu'elle est constituée au plan du macrocosme et du microcosme par les éléments des différents niveaux de réalité. Ces éléments sont identifiés dans le Timée comme étant le démiurge, les formes intelligibles et le milieu spatial. Cette structure ontologique appliquée au macrocosme donne l'âme du monde et le corps du monde tandis qu'appliquée au microcosme elle donne la réalité de l'homme. Reste un résidu, la nécessité, qui échappe au contrôle de la raison démiurgique. D'où nous obtenons les grandes articulations de ce commentaire systématique du Timée: 1. Le Démiurge (pp. 29-106), 2. Le monde des formes intelligibles (pp. 107-173), 3. Le milieu spatial (pp. 177-266), 4. L'âme du monde (pp. 267-354), 5. Le corps du monde (pp. 355-412), 6. L'homme (pp. 413-465), 7. La nécessité (pp. 469-513). Pourquoi maintenant le Même et l'Autre? C'est que chacun des éléments de la structure ontologique peut être considéré sous le triple aspect fondamental de l'être que Platon avait déjà explicité dans le Sophiste, c'est-à-dire la substance, le même et l'autre. Ajoutons à cela que chacune des sections de l'ouvrage comprend trois étapes : la première consiste dans une analyse rigoureuse du texte, la seconde poursuit l'examen méthodique des différentes interprétations dans l'ancienne académie, le moyen platonisme et le néo-platonisme, et la troisième essaie d'établir des rapports entre les interprétations anciennes et les interprétations plus récentes du XIXe siècle et du XXe siècle. Comme on peut facilement le constater, le commentaire systématique du 
Timée s'ouvre en réalité sur une véritable histoire du platonisme ancien et du platonisme moderne.

Notre première remarque portera sur les rapports entre le Timée et ce que l'on a couramment appelé les doctrines non écrites de Platon. L. Brisson refuse l'hypothèse d'une doctrine non écrite de Platon parce que celle-ci ne s'appuie, en définitive, que sur deux passages d'Aristote : celui de Physique, 209b11-16 et celui du De Anima, 404b16-30 (pp. 221-226, pp. 276-290), qu'il refuse d'ailleurs de considérer comme parallèles ou encore de relier au $\pi \epsilon \rho i \tau \dot{\alpha} \gamma \alpha \theta \sigma \tilde{v}$ d'Aristoxène (p. 224). Cette hypothèse, selon L. Brisson, aurait été faite pour donner un sens au témoignage d'Aristote sur la théorie des nombres-idéaux qui présente une doctrine fondamentalement différente de celle des dialogues. Mais celle-ci lui semble superflue pour deux raisons : d'abord parce qu'Aristote est plus philosophe qu'historien de la philosophie, ensuite parce qu'il critique souvent en commun Platon, Speusippe et Xénocrate et que cette théorie des nombreux-idéaux serait davantage celle de Xénocrate que celle de Platon (p. 225). L. Brisson préfère donc s'en tenir à la doctrine écrite de Platon dans son interprétation du Timée, ce qui a pour conséquence de faire d'Aristote un très mauvais interprète de Platon.

Ce n'est pas le lieu ici d'entrer dans tous les détails de cette controverse, mais nous aimerions seulement souligner le fait que cette hypothèse d'une doctrine non écrite de Platon est à l'origine de beaucoup de travaux contemporains qui ont renouvelé notre conception du platonisme et qu'on ne saurait la rejeter d'un revers de la main. Cette hypothèse ne s'appuie pas seulement sur la

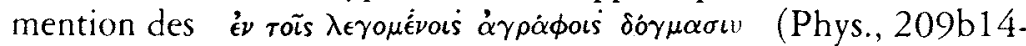

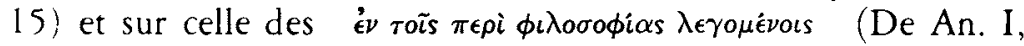
404 b19), mais sur une vaste documentation dont le noyau comprend la Métaphysique d'Aristote, son traité perdu Sur les Idées que nous connaissons par Alexandre d'Aphrodise ainsi que son traité Sur le Bien dont les trois livres ont servi de base à un long passage de Sextus Empiricus dans son ouvrage Contre les Mathématiciens (X, 248-283). Au début du siècle, L. Robin avait attiré l'attention des platonisants sur cette vaste documentation d'Aristote en prenant soin de distinguer, selon la suggestion même de Met. M, 4, 1078b9-12, les exposés d'Aristote sur la théorie des Idées sous sa forme primitive et les exposés sur la dernière théorie 
des nombres-idéaux. Celle-ci était présentée par Aristote sous la formulation d'une théorie de l'Un et de la Dyade du Grand et du Petit que l'on ne rencontre nulle part dans les dialogues et qu'Aristote attribuait explicitement non à Speusippe ou à Xénocrate, mais à Platon lui-même ${ }^{s_{0}}$. L. Brisson appuie son refus des doctrines non écrites de Platon sur deux célèbres ouvrages de $\mathrm{H}$. Cherniss dont l'influence d'ailleurs demeure marquante sur sa propre conception générale du Platonisme (Aristotle's criticism of Plato and the Academy, 1944, The Riddle of the early academy, 1945). Mais les positions de $\mathrm{H}$. Cherniss ont reçu tout récemment une critique sévère de la part de J.N. Findlay (Plato The Written and Unwritten Doctrines, 1974), selon lequel les doctrines écrites de Platon ne prennent vraiment tout leur sens qu'à la lumière des doctrines non écrites. ${ }^{51}$ Entre-temps, les études de K. Gaiser (Platons Ungeschriebene Lebre, 1963, Platons Meno und die Akademie, 1964) et celles de H.J. Krämer (Arete bei Plato und Aristotles, 1959, Über den Zusammenhang von Prinzipienlebre und Dialektik bei Platon, 1966), que la critique saluait comme une nouvelle dimension du platonisme (A. Solignac, P. Aubenque, Archives de Philosophie, 28 (1965) 251-265), ou encore comme une redécouverte de Platon (J. Pépins, Preuves, 18 (1968) 76.84), nous révélaient l'importance des doctrines non écrites dans l'interprétation des dialogues de Platon et surtout de sa dernière philosophie's. On ne saurait donc renoncer aussi facilement que le fait L. Brisson à l'hypothèse de l'existence de doctrines non écrites de Platon dans le contexte actuel des études platoniciennes. Nous avons conscience que si cette hypothèse s'avérait vraie, l'auteur devrait reprendre une bonne partie de son interprétation du platonisme dans l'ancienne académie ainsi que sa lecture des témoignages d'Aristote sur la philosophie de Platon et en particulier sur la théorie des Idées.

50. RoBIN, L., La Théorie Platonicienne des Idées et des Nombres d'après Aristote, (1908), Hildesheim, (ieorg. Olms, 1963. On trouvera en pp. 635.654 une longue discussion sur l'attribution de la théorie de l'Un et de la Dyade indéfinie à Platon et les passages d'Aristote dans lesquels la théorie est explicitement attribuée à Platon.

51. On lira surtout ici les pp. 19.25 de l'introduction ainsi que l'appendice II consacré entièrement à une critique des positions de $\mathrm{H}$. Cherniss sur l'hypothèse des doctrines non écrites (pp. $455-473$ ).

52. Nous nous permettons de signaler ici le recueil d'études édité par J. Wippern, Das Problem der Lngeichriebenen Lebre Platons, Darmstadt, 1972 qui présente les contributions les plus valables sur le sujet de 1929 à 1966 , ainsi qu'une ample bibliographie. 
C'est aussi sous l'influence de H. Cherniss (The pbilosophical economy of the theory of ideas, 1936) et sur le refus des doctrines non écrites de Platon (p. 111) que L. Brisson nous donne son interprétation de la théorie des formes intelligibles dans le Timée et d'une façon plus générale dans la philosophie de Platon (pp. 111-136). Ce qui frappe dans cette interprétation, c'est la persistance d'une même théorie des Idées dans les dialogues de maturité et dans les dialogues de vieillesse (p. 126), ce que nous appellerions une conception statique de la théorie des Formes intelligibles. Il nous semble difficile d'admettre que Platon n'ait pas évolué sur ce point vers une mathématisation progressive de sa théorie des Formes et qu'il n'ait pas cherché à la dégager du mysticisme de la maturité. Sur ce point, il se pourrait que les doctrines non écrites puissent nous instruire et nous aider à comprendre les modifications profondes que subissent les Formes dans la dernière philosophie de Platon. Â ce titre, il ne nous semble pas que la méthode de divison dans le Sophiste puisse être considérée purement comme un "procédé heuristique destiné à la réminiscence ", (p. 125) mais bien plutôt comme une nouvelle dialectique qui travaille sur les fonctions logiques des Formes-Genres et qui peut se passer du processus de réminiscence largement exploité dans les dialogues de maturité. Ces remarques, nous le savons, pourraient nous entraîner fort loin. Qu'il nous suffise de dire que nous serions porté à privilégier une conception évolutive de la théorie des Formes intelligibles dans le sens d'une mathématisation progressive et qui serait assez différente de la théorie des Formes de la maturité. Et cela correspondrait à la réputation de philosophegéomètre que Platon s'était acquise dès l'antiquité. Quoiqu'il en soit de ces remarques sur lesquelles il nous est présentement impossible d'exercer toute l'acribie qu'elles demanderaient, nous devons reconnaître que le commentaire du Timée de L. Brisson est si riche en érudition, en spéculation philosophique et en perspectives historiques sur le platonisme qu'il exigerait beaucoup plus que les quelques paragraphes que nous venons de lui consacrer ${ }^{53}$.

53. On trouvera une étude critique sur l'ouvrage de L. Brisson faite par Jean Trouillard, ds. Pbiloseppliquer, 2 (1975) 287.299 . 
D'une façon générale, on peut dire que la contribution canadienne aux études platoniciennes reflète la diversité des méthodes d'approche du texte platonicien chez les platonisants contemporains. Les uns mettent l'accent sur l'approche philologique (Robinson, Paquet), d'autres sur l'approche philosophique (Seligman), ou sur l'analyse purement conceptuelle (Allen, Shiner, Gallop), ou encore sur l'approche historique (Brisson). Cependant, ces méthodes ne sont pas utilisées en exclusivité de sorte qu'il est difficile de classifier d'une façon très nette les platonisants canadiens. Il nous est apparu, toutefois, que les platonisants d'expression française se relient facilement à la tradition continentale qui privilégie l'approche philologique, historique et philosophique $\mathrm{du}$ texte platonicien (Paquet, Brisson) tandis que les platonisants d'expression anglaise s'inspirent plus ou moins de la tradition analytique en valorisant l'analyse conceptuelle. Les représentants de cette tendance sont Gallop, Allen, Shiner. Quant à Robinson et à Seligman, ils nous sont apparus plus proches de la tradition continentale que de la tradition analytique.

\section{Université d'Ottawa}

Appendice

Sans prétendre à l'exhaustivité, nous avons recueilli un certain nombre d'articles sur Platon publiés par des collègues canadiens entre les années 1960-1976 dans diverses revues philosophiques nationales et internationales. Le talon d'Achilie d'une telle bibliographie, on en conviendra, réside dans l'identification de l'auteur comme canadien. Nous prenons ce terme dans le sens large d'un individu qui travaille présentement dans une institution canadienne. Pour ce travail, nous avons dépouillé le Pbilosopher's Index (1967-1976) et le Répertoire Bibliographique de Louvain (19601976). Nous faisons suivre cette bibliographie de la liste des auteurs cités et de leur lieu de travail pour aider les chercheurs en études platoniciennes à pouvoir plus facilement se consulter entre eux sur des problèmes qui les préoccupent. Pour établir le lieu de travail nous avons utilisé la liste des professeurs de philosophie enseignant au Canada faite par I'Association Canadienne de Philosophie (1976-1977), ainsi que les indications fournies dans la revue où l'article est publié. Nous sommes conscient de la difficulté d'être exhaustif dans ce genre de travail et nous prions les auteurs que nous avons malheureusement oubliés de bien vouloir nous en excuser et de nous en avertir pour être en mesure d'apporter les corrections nécessaires dans un prochain numéro de la revue. 
GAUDRON, E., "Sur l'Objet du Sophiste, " ds. Laval Théol. et Philos., 16 (1960) 70-93, RIST, J.M., "The Order of the Later Dialogues of Plato," ds. Phoenix, 14 (1960) 207.221, WOODMAN, R.S., "Shelley's Changing Attitude to Plato, "ds. Journ. of the Hist. of Ideas, 21 (1960) 497-510, ALLEN, R.E. "The Socratic Paradox, "ds. Journ. Hist. Ideas, 21 (1960) 256-265, GALLOP, 1.. "Justice and Holiness in Protagoras," ds. Phronesis, 6 (1961) 86-93, ALLEN, R.E., "The Argument From Opposites In Republic V, "ds. Rev. of Metaphyrics, 15 (1961) 325-335, RIST, J.M.. "The Neoplatonic (Ine and Plato's Parmenides, "ds. Trans. and Proc. of the Am. Pbilol. Ass. 93 (1962) 389-401, RIST, J.M., "The Parmenides Again," ds. Pboenix, 16 (1962) $1-14$ GAli.OP. D., "Plato and the Alphabet," ds. Pbilos. Rev., 72 (1963) 364-376, RIST, J.M., "Plotinus and the Daimonion of Socrates, "ds. Pboenix, 17 (1963) 13-24, LAFRANCE, Y., "A propos d'une thèse de Kesters, " ds. Dialogue 2 (1963) 83-95, GAUDRON, E., "La Théorie des Idées dans le Phédon, "ds. Laval Théol. et Philos, 20 (1964) 50-73, ODEGARD, D., "Essences and Discovery; Plato, Locke and Leibnitz," ds. Dialogue 3 (1964) 219-234, RIST, J.M., "Equals and Intermediates in Plato," ds. Pbronesis, 9 (1964) 27-37, GALLOP, D., "The Socratic Paradox in the Protagoras, "ds. Pbronesit, 9 (1964) 117-129. ALLEN, R.E., "The Interpretation of Plato's Parmenides : Zeno's Paradox and the Theory of Forms, "ds. Journ. of the Hist. Pbilor. 2 (1964) 143.157. SAVAN, D., "Self-Predication in the Protagoras, "ds. Phronesis,9 (1964) 130-135. RIST, J.M., "Neopythagoreanism and Plato's Second Letter, " ds. Phronesis, 10 (1965) 78-82. SPARSHOTT, F.E., "Socrates and Thrasymachus, " ds. The Monist, 50 (1966) 421-459, WOLFE, J., "A Note on Plato's "Cyclical Argument " in the Phoedo ", ds. Dialogue 5 (1966) 237-238, ALLEN, R.E., "A Note on the Elenchus of Agathon Symposium 199c-201c, ds. The Monist. 50 (1966) 460-463, RIST, J.M., "Knowledge and Value in Plato ", ds. Phoenix, 21 (1967) 283-295, ROBINSON, T.M., "Demiurge and World Soul in Plato's Politicus ", ds. Americun foum. of Plsilol. 88(1967) 57.67. ROBINSON, T.M., "Soul and Immortality in Republic $\mathrm{X}$ ".ds. Phronesis. 12 (1967) 147-151, HARRISSON, E.L., "Plato's Manipulation of Thrasymachus ". ds. Ploenix, 21 (1967) 27-39, SPARSHOTT, F.E., "Plato as Anti-Political Thinker ", ds. Ethicr. 77 (1967) 214-219, PHILIP. J.A., "False Statement in the Sophist ", ds. Trans. and Proc. of the Amer. Plilol. Ass. 99 (1968) 315-329, PHILIP, J.A., "The Apocrapha of Platos Suphist "ds. Plonenix, 22 (1968) 289-299, ROBINSON, T.M., "The Nature and Significance of the Argument for Immortality in the Phaedrus ", ds. Apeiron, 2 (1968) 12-18, WHITTAKER, J., "The Eternity of the Platonic Forms ", ds. Pbronesis, 13 (1968) 131.144, WHITTAKER, J., "Thimaeus 27d5ss.", ds. Phoenix, 23 (1969) 181-186, LAFRANCE, Y., "La Problématique morale de l'opinion dans le "Gorgias" de Platon", ds. Rev. Pbil. de Louvain, 67 (1969) 5-29, CAl.VERT. B.. "Forms and Flux in Plato's Cratylus", ds. Pbronesis, 15 (1969) 26-47. DORTER. K. "The Significance of the Speeches in Plato's Symposium ", ds. Phil. Rhet., 2 (1969) 215-234. ROBINSON, T.M. "Phaedo 70c: An Error and Explanation ", ds. Dialogue, 8 (1969) 124-125. PHILIP. J.A., "The Megista Gene of the Sophistes ", ds. Phoenix, 23 (1969) 89.103, DYBIKOWSKI, J.C., "Mixed and False Pleasure in the Philebus : a Reply ", ds. Philos. Quart. 20) (1970) 244-247. PHILIP. J.A., "The Platonic Corpus ", ds. Pboenix, 24 (1970) 296-30\%, BRISSON. L., "L.'Instant, le Temps et l'Éternité dans le Parménide (155e.157d) de Platon "ds. Dialogue, 9 (1970) 389-396, BRISSON, L., "De la Philosophie à l'Épopée : Le Critias de Platon ". ds. Rev, Metaph. et Morale, 75 (1970) 402-438, DORTER, K., "The Dramatic Aspect of Plato's Phaedo ", ds. Dialogue, 8 (1970) 564-580, DYBIKOWSKI, J.C., "False Pleasure and the Philebus, ds. Phronesis, 15 (1970) 147-165, ALLEN, R.E., "The Generation of Numbers in Plato's Parmenides", ds. Class. Philol., 65 (1970) 30-34, SHINER, R.A., "SelfPredication and the "Third Man "Argument", ds. Journ. of the Hist. of Philos., 8 (1970) 371. 386. SPARSHOTT, F.E., "Five virtues in Plato and Aristotle", ds. The Monist, 54 (1970) 4065. THOMAS, J.E., "A Re-Examination of the Slave-Boy Interview ", ds. Laval Théol et Pbilos., 26)(1970) 17.29. THORTON, M.T., "Knowledge and Flux in Plato's Cratylus ", ds. Dialogue, 8 (1970) 581.591. GOOCH, P.W., "Vice is Ignorance' : The Interpretation of Sophist 226a-231b, ds. Phnenix. 25 (1971) 124.133, DORTER, K., "Imagery and Philosophy in Plato's Phaedrus ", ds. Journ. of the Hist. of Philos. 9 (1971) 279-288, LAFRANCE, Y., "Le Platon de Gilbert Ryle ", ds. Rer. Philor de Louvain, 69 (1971) 337-369, LAFRANCE, Y., "La Psychologie de Platon selon Y. Brès ", ds. Dialogue, 10)(1971) 134-145, LAFRANCE, Y., " Communication et violence dans le dialogue socratico-platonicien ", ds. La Communication. Actes du XVe Congrès des Sociétés de Philosophie de langue française, 1971, p. $101-105$, PHILIPPOUSIS, J.. " La gnoséologie de Platon selon la République : connaissance et dialectique ", ds. La Communication, of. cit.. 1971. p. 90-95. GIROIIX, L., "Bergson et la conception du Temps chez Platon et Aristote ", ds. 
Dialogute. 10 (1971) 499-503. SPARSHOTT, F.E., "The Thruth about Gods and Men", ds. Dialogue. 10 (1971) 3.11. WOODBURY, L., "Socrates and Archelaus", ds. Phoenix, 25 (1971) 209-309. DORTER. K.. "Equality, Recollection and Purification, ds. Phronesis, 19 (1972: 198-218. PFEIFFER. W.N. "True and False Speach in Plato's Cratylus, 385b-c ", ds. Cin. Jomm of Pilos. 2 (1972) 87.104, SHINER, R.A., Soul in Republic X. 611 ", ds. Apeiron, 6 (1972! 23-30. Al_LEN, R.E., "Law and Justice in Plato's, Crito ", ds. Journ. Philol., 69 (1972) 557.567. DYBIKOWSKI, J. "Critical Notice of T.M. Robinson Plato's Psychology ", ds. Can. Journ. of Pbilos. 3 (1973) 131-142. DORTER, K., "The Ion : Plato's, Characterization of Art ", ds. Joum. Aestls. and Art. Crit., 32 (1973) 65-78. LAFRANCE, Y., "Amour et Violence dans la Dialectique platonicienne ". ds. Dialogue, 12 (1973) 288-308, WHITTAKER. J., "Textual Comments on Timaeus. 27c-d ". ds. Phoenix, 27 (1973) 387-391. PAQUET, L., "Platon ou l'Amour Philosophe ". ds. La Plitosophie et les Philosophes, Montréal, Bellarmin, 1973, Vol. I, pp. 27-43, FREIRE. A.. "Eros platonicien et agapê chrétienne", ds. An. Un. Bucuresti, 22 (1973) 23-35, DORTER, K., "Socrates Refutation of Thrasymachus and Treatment of Virtue ", ds. Philos. Rhet., 7 (1974) 25.46. DYBIKOWSKI, J., "Socrates, Obedience and the Law : Plato's Crito ", ds. Diahegue. 13 (107.4) 519-535, PANAGIOTOU, S.. "Relations and Infinite Regress in Plato", ds. Dialugue. $13(1974) 537.543$, THOMAS, J.E., "On the Duality of Socrates' What-is-XQuestion ". ds. Laral Thérl. et Philor. 30)(1974) 21-27. LAFRANCE, Y., "De la Nature à la Culture selon Platon ".ds. Actes du XVIe Congrès des Sociétés de Pbilosophie de Langue Françatse. Reims, 197/4. pp. 9)-101, KUBARA, M., "The Logic of Virtue in the Republic "ds. Can. Journ. of Pliths. Suppl. I (1974) I I-27, ALLEN, R.E., "Unity and Infinity : Parmenides 142b-145a". ds. Re', Metaph. 27 (1974) 699-725, LERO(IX, G., " Metaphysique et Politique chez Platon ". ds. Pbilowphies de la Cité, Montréal, Bellarmin, 1974, pp. 11-38, LEROUX, G., "Platon. La Médiation du Regard ", par L. Paquet, ds. Pbilosopbiques, 2 (1975) 253-285 PELLETIER. F.J.. "Incomptability' in Plato's Sophist ", ds. Dialogue, 14 (1975) 143-146. LAFRANCE. Y.. "Platonisme et Pensée Contemporaine ", ds. Dialogue, 14 (1975) 147-158. PANAGIOTOU, S.. "Lysias and the date of Plato's Phaedrus", ds. Mnemosyne, 28 (1975) 388-398. RIST. J.M.. "Plato's Earlier Theory of Forms". ds. Phoenix, 29 (1975) 336-357, CALVERT, B.. "Plato and the Equality of Women", ds. Phoenix, 29)(1975) 231-243 HOGAN, R.A.. "Soul in the Charmides: an examination of T.M. Robinson's Interpretation", ds. Phil. Res. Arch. 2, no 1095 , 1976\%. DORTER, K., "Socrates on life, death and suicide ", ds. Laval Théol. Philor. 32 (1976) 23-41. PHIL.IPPOUISIS. J.. "Heidegger and Plato's Notion of Truth", ds. Dialogue. 15 (1976) 502-504. LAFRANCE. Y., "L usage de l'analogie dans l'interprétation de Platon", ds. Dialogue. $15(1976$ 282-289. 7\%HOMAS. J.E., "Models for Muddles at Meno 75a-77a, ds. Neu Scbolas., 50) (1976! 193-203.

Allen, R.E. (Iniversity of Toronto) Brisson. L. (C..N.R.S. Paris) (it)ert. 13. (l Iniversity of (iuelph) Dorter. K. (Iniversity of (iuelph) D)bikowski. J. (I Iniversity of British Columbia) Gaudron, E. (Iniversité Laval) (iallop, 1). (Trent Iniversity) (iiroux. I. (Iniversité de Sherbrooke) (iooch, P.W. (Iniversity of Toronto) Kubara. M. (Iniversity of Lethbridge) Lafrance. Y'. (Iniversité d'Ottawa) Leroux. (i. (tIniversité du Québec à Montréal) (Begitrd. 1). (Iniversity of (iuelph) Panagiotou. S. (Iniversity of Guelph) Paquet. I. (Inniversité dottawa)
Pelletier, F.J. (University of Alberta) Pfeiffer. W.N. ([Iniversity of Waterloo) Philip. A. (IIniversity of Toronto) Philippousis, J. (Collège Dawson. Montréal) Rist. J.M. (Iniversity of Toronto) Robinson, T.M. (I Iniversity of Toronto) Savan, 1). (Iniversity of Toronto) Seligman. P. (University of Waterloo) Shiner. R.A. (University of Alberta) Sparshott. F.F. (I Inversity of T'oronto) Thorton, J.E. (I Iniversity of Toronto) Thomas. J.f. (Mc Master (Iniversity) Whittaker. J. (Menorial (Iniversity) Wordbury. L.F. (llniversity of 'Toronto) Woxdmin. R. (i. (Western Otario) Wolfe J. (Carleton University) 